Retour sur le libéralisme conservateur de Wilhelm Röpke

A reappraisal of Wilhelm Röpke's conservative liberalism

\title{
Raphaël Fèvre
}

\section{(2) OpenEdition}

\section{Journals}

Édition électronique

URL : http://journals.openedition.org/ress/3332

DOI : $10.4000 /$ ress.3332

ISBN : $1663-4446$

ISSN : 1663-4446

Éditeur

Librairie Droz

Édition imprimée

Date de publication : 15 novembre 2015

Pagination : 147-190

ISSN : 0048-8046

Référence électronique

Raphaël Fèvre, «Retour sur le libéralisme conservateur de Wilhelm Röpke », Revue européenne des sciences sociales [En ligne], 53-2 | 2015, mis en ligne le 15 novembre 2018, consulté le 19 avril 2019 URL : http://journals.openedition.org/ress/3332 ; DOI : 10.4000/ress.3332 


\title{
RETOUR SUR LE LIBÉRALISME CONSERVATEUR DE WILHELM RÖPKE
}

\author{
RAPHAËL FÈVRE \\ Université de Lausanne, Centre d'études interdisciplinaires Walras-Pareto \& \\ Université Paris I, Centre d'économie de la Sorbonne \\ raphael.fevre@gmail.com
}

\begin{abstract}
Résumé. L'objet de cet article est double. C'est, d'abord, une reconstruction du discours décliniste de Wilhelm Röpke [1899-1966], de son articulation et de sa singularité. C'est ensuite une explicitation des prétentions théoriques de l'auteur à l'aune de l'analyse historique qui est la sienne. L'article fait ainsi apparaître les préoccupations fondamentalement morales de Wilhelm Röpke et leurs implications au niveau de son libéralisme tant économique que politique. On conclut alors que si ces dernières mettent en lumière la cohérence d'une œuvre orientée vers une réforme sociétale ambitieuse, elles n'en restent pas moins impuissantes à associer de manière rigoureuse une logique interventionniste libérale avec un contenu conservateur social.
\end{abstract}

Mots-clefs: dépression, ordolibéralisme, pouvoir économique et politique, Wilhelm Röpke.

\begin{abstract}
The aim of this paper is twofold. First, it is a reconstruction of Wilhelm Röpke's [1899-1966] declinist discourse, its articulation and its singularity. It is subsequently an explanation of the author's theoretical claims in the light of his historical analysis. Thus the article reveals the primarily moral concerns of Wilhelm Röpke and their implications for his economic and political liberalism. It then concludes that, if this moral background sheds light on a work which actually aims for an ambitious societal reform, it remains unable to rigorously associate a liberal interventionist logic with a social conservative content.
\end{abstract}

Keywords: economical and political power, ordoliberalism, recession, Wilhelm Röpke.

Codes JEL: B25, B31, F02. 
INTRODUCTION

Le désordre social, c'est, pour l'économiste, une équation qui n’est pas satisfaite, mais pour le sociologue, une civilisation qui meurt dans les troubles d'une douloureuse agonie. Jacques Rueff (1967 [1945], p. 598)

Ces mots de Jacques Rueff résument l'œuvre d'une vie: celle de Wilhelm Röpke, figure centrale de l'ordolibéralisme allemand. Ses travaux, point de rencontre entre l'économie, la sociologie et la philosophie de la culture, offrent une étude à la fois négative et positive du libéralisme, basée sur l'examen de son cheminement dans l'histoire de l'Occident. En ce sens, il est également - et peut-être avant tout - un penseur du déclin, mariant analyse historique et économie politique. L'ambition de cet article est de reconstruire cette analyse historique à la hauteur de l'ensemble de l'œuvre de Röpke, en mettant en exergue certaines catégories à même d'éclairer ses prétentions positives. Il s'avère à notre sens indispensable d'expliciter sa lecture historique afin d’interpréter avec précision son approche du libéralisme. En effet, son explication décliniste ne porte pas seulement sur des causes socio-politiques et économiques, mais est fondamentalement réductible à une critique morale et philosophique. En ce sens, on montrera qu'il propose une réhabilitation originale du libéralisme économique sur une base morale conservatrice ${ }^{1}$. Tout en rendent intelligible le caractère total de son interventionnisme, on souligne également des incohérences aux regards des politiques économiques qu’il préconise. Ce faisant, on retrace la généalogie de certains aspects qui marquent l'unité de l'ordolibéralisme allemand (constructivisme étatique, rôle des experts, politiques économiques constitutionnelles). Mais la singularité fondamentale de Röpke, eu égard à ses sérieuses réserves morales et sociales quant à la diffusion d’un ordre économique basé sur la concurrence, n’est pas pour autant négligée.

I On entend ici «conservateur» dans le sens de critique de la modernité - en tant que dynamique socioculturelle - et non directement de préservation du statu quo. Cette critique repose essentiellement sur une volonté de préserver l'autonomie des groupes de proximités, une autorité et une hiérarchie qui émane de la tradition et de la religion, certaines valeurs tutélaires inaliénables. Le déroulement de l'article aide à étoffer le contenu du terme et pourquoi on l'attribue à Röpke. Sur le conservatisme des milieux germaniques du premier Xx siècle, voir Habermas, 1989; Mannheim, 2009 (1984) et Puhle, 1978. 
Positionner les travaux de Wilhelm Röpke dans le panorama de l'histoire de la pensée économique n’est pas chose aisée. Sa volonté de penser en système nourrit une œuvre féconde, mais qui reste trop souvent à des niveaux de généralités préjudiciables, particulièrement au plan analytique. Déplorant l'effacement de l'économie en tant que science morale, certains commentateurs signalent néanmoins que ses positions évoquent - tant sur le fond que sur la forme - celles d’Adam Smith (Hutchison, I979; Sally, I996; Gregg, 2010, p. I2-I3 et p. 173-176; Bonefeld, 2013).

Wilhelm Röpke est né près de Hanovre en $1899^{2}$ au sein d'une famille protestante très attachée à la ruralité et ses traditions. Il obtient son doctorat d'économie à l'université de Marbourg en 1921. En parallèle à son parcours dans différentes universités allemandes (Jena, Graz, Marbourg), il conseille le ministère de l'Économie du gouvernement d'Heinrich Brüning (Boarman, 2000). Mais lors de l'avènement au pouvoir d’Hitler en 1933, privé du droit d'exercer comme professeur en raison de ses «opinions non conformes » (Bilger, 1964, p.94), il s'exile en Turquie pour enseigner à l'université d'Istanbul. Il y demeure jusqu'en 1937 avec son ami et collègue Alexander Rüstow, lequel a une influence décisive sur sa pensée, influence d’ailleurs réciproque. Röpke obtient finalement la chaire de Relations économiques internationales à l'Institut universitaire des hautes études internationales de Genève ; poste qu'il occupe jusqu'à son décès en 1966. C'est dans ce cadre qu'il participe à la fondation de la Société du Mont-Pèlerin, dont il sera président de 196r à 1962, mais qu’il quittera ensuite, mécontent de la direction prise

2 II partage cette année de naissance avec le principal promoteur de la pensée libérale du $x x^{e}$ siècle: Friedrich Hayek. II y aurait beaucoup à dire sur les parcours personnels et intellectuels des deux auteurs qui s'estimaient mutuellement malgré leurs différends. Hayek reconnaît en Röpke «leplus actif et le plus productif » des libéraux allemands; il ajoute: «si l'existence d'un mouvement néo-libéral est connue largement, à tout le moins dans les pays de langue allemande, au-delà même des milieux professionnels, le mérite en revient sans aucun doute essentiellement à lui » (cité par Bilger, 1964, p. 5). Sans s'interdire toute référence à Hayek, la posture comparatiste n'est clairement pas l'objet de cette étude. Nous ne pouvons que renvoyer le lecteur intéressé aux travaux de l'historien Jean Solchany (2015), qui signe une imposante biographie intellectuelle (pour une autre biographie, voir Hennecke, 2005). Sur des rapprochements doctrinaux, on pourra consulter les articles de Stefan Kolev (20I0) ou Manuel Wörsdörfer (20II). 
par l'institution (Steiner et Walpen, 2006, p. 98 ; Burgin, 20I2, p. I43-I46). Outre ses fonctions académiques et son imposant travail journalistique de vulgarisation des idées libérales, Röpke a conseillé des personnalités politiques de premier plan comme Ludwig Erhard et Konrad Adenauer, architectes du «miracle économique » allemand (Goldschmidt, 2006, p. 962).

Malgré une formation dont l'empreinte historiciste est manifeste et une proximité avec les idéaux socialistes durant sa jeunesse, Wilhelm Röpke se révèle être un fervent défenseur des thèses marginalistes (en particulier les travaux de l'école autrichienne d'économie) ainsi qu'un critique incessant de l'économie collectiviste.

Très peu de travaux lui sont exclusivement dédiés. Cependant, son parcours transnational et ses compétences variées font de lui un «idéal-type de l'intellectuel néolibéral» (Solchany, 2oI3, p. 6), et à ce titre un objet qui donne à penser la (re)production du libéralisme au xx siècle. Les études qui abordent la pensée de Wilhelm Röpke traitent principalement de son apport à l'ordolibéralisme allemand et à l'économie sociale de marché (Friedrich, 1955; Oliver, 1960; Bilger, 1964; Grossekettler, 1989 et 1994; Gerber, 1994 et 1998; Sally, 1996) ou plus récemment à la «nébuleuse » néolibérale (Doering, 2006 ; Jackson, 20ı ; Audier, 2012; Siems et Schnyder, 2012; Coleman, 2013). Elles se concentrent également sur les liens entre son approche sociologique de l'économie et la pensée chrétienne (Campbell et Foshee, 1997; Leen, 2003; Ycre, 2003; Doering, 2006); ou encore sur l'influence qu'il a exercé en tant qu'intellectuel libéral à la Société du Mont-Pèlerin (Steiner et Walpen, 2006; Ptak, 2009), ainsi qu'auprès des décideurs politiques lors de la reconstruction de l'Allemagne d’après la Seconde Guerre mondiale (Nicholls, 1994; Commun, 2003b).

Trois thèmes principaux permettent de périodiser ses contributions ${ }^{3}$ suivant deux étapes. Il s'est d’abord concentré sur la théorie des cycles (1933b, I936a, 1936b), notamment avec une approche de stimulation proto-keynésienne (voir Klausinger, 1999). Dans un second temps, c’est la crise générale du

3 Nous ne suivrons pas directement le découpage opéré par Samuel Gregg dans la monographie qu'il consacre à l'auteur allemand (2010, p.9): cycle des affaires; welfare state, inflation et chômage; relations économiques internationales. En effet, en privilégiant les travaux 
monde occidental qui l'occupera, à laquelle il tentera d'apporter des réponses (1942, 1945 [1942], 1946 [1944], 1959a). Face à l'essor de la pensée keynésienne et la diffusion du Welfare State, il mènera en parallèle une critique d'une forme d’interventionnisme étatique à son sens fallacieux (1957, 196I [1958], 1969).

Notre travail portera plus spécifiquement sur la partie centrale du corpus de l'auteur : celle où il aborde la Grande Dépression comme l'acmé d'un déclin philosophique, culturel, social et économique entamé un siècle plus tôt. Chez Röpke, les manifestations économiques actuelles ne sont que les symptômes d'une «maladie organique profonde» (Röpke, 1942, p. 2, notre traduction) qui prend racine au XIX ${ }^{\mathrm{e}}$ siècle.

À ce titre, une première partie traitera des causes du déclin identifiées par Röpke comme étant de natures économique et socio-politique, mais fondamentalement réductibles aux domaines moral et philosophique. Une attention particulière sera portée à l'articulation et l'enchaînement des symptômes ainsi qu'à l'exemple des différentes trajectoires nationales qu'il évoque. Dans une seconde partie, nous présenterons les solutions institutionnelles soutenues par Röpke pour remédier à ces phénomènes et, notamment, sa nouvelle approche «scientifique » du libéralisme - le Tiers Chemin - passant par l'interdisciplinarité. Un gouvernement fédéral élitiste et anti-pluraliste doit remédier à la crise du système démocratique. Dans ces conditions, l'État fort se porte garant d'un cadre juridico-institutionnel via un interventionnisme libéral à même de dépasser le libéralisme historique dégénéré. 


\section{LE DÉCLIN ÉCONOMIQUE ET SOCIAL DU MONDE OCCIDENTAL Une interprétation morale et philosophique}

Nous intéressent ici moins les symptômes finaux - c'est-à-dire la crise ${ }^{4}$ - que l'analyse de ses causes profondes; ce qui revient à mettre à jour l'analyse du déclin opérée par Röpke sur le long terme. Cette analyse du déclin peut être ramenée à trois domaines : économiques et socio-politiques, symétriquement, et moraux et philosophiques, ultimement. Ce découpage a du sens dans la mesure où les facteurs moraux sont premiers tout en étant le vecteur principal de facteurs sociaux et économiques (1942, p. 2 ; 1945 [1942], p. 24), mais doit être néanmoins sérieusement nuancé. Comme l'écrit Röpke,

la crise spirituelle et morale d'une part et la crise politique, sociale et économique (sociologique) de l'autre [...] se pénètrent étroitement et s'influencent mutuellement. Car la société, dans toutes ses parties et sous tous ses aspects, forme toujours un ensemble dans lequel l'interdépendance et la coordination ne cessent d'affirmer leurs droits (1945 [1942], p. 18).

Aussi, quoique les causes socio-politiques et économiques soient corrélatives, elles seront cependant dissociées pour clarifier l'exposés. En remontant vers les causes ultimes, on montrera que Röpke, en définitive, ramène les causes sociales, politiques et économiques au seul domaine moral et philosophique.

4 Pour Röpke, la Première Guerre mondiale fait basculer le monde occidental d'une période de déclin à la crise effective, marquée pas des bouleversements structurels subséquents. De tels phénomènes - comme l'hyperinflation allemande, la crise financière de 1929 et l'effondrement du système monétaire international de l'étalon-or -, dépassent la simple oscillation cyclique. Plus qu'un phénomène économique et quantitatif, la crise s'exprime aussi - et surtout - dans le domaine spirituel (baisse de l'importance du religieux), social (chômage de masse) et politique (montée du nationalisme et du collectivisme).

5 L'article procède donc à une reconstruction rationnelle des arguments de Röpke, notamment afin de souligner la symétrie avec ses propositions de réformes. Pour une présentation - à la fois plus compréhensive et contextuelle - entièrement consacrée à la lecture que propose Röpke de la crise du $x x^{e}$ siècle comme un enchaînement causal du libéralisme historique menant inéluctablement à la planification économique, voir Fèvre (20I5). 


\section{I.I. LES CAUSES ÉCONOMIQUES}

\section{Concentration, division du travail et machinisme}

Participant à la séance «Le déclin du Libéralisme est-il dû à des causes endogènes? » au colloque Walter Lippmann ${ }^{6}$ - organisé à Paris en 1938 -, Wilhelm Röpke est incapable de proposer une réponse claire à cette interrogation. La même incertitude va parcourir l'ensemble de ses travaux en la matière. Deux positions vont se faire face lors de cette discussion qui divise le camp libéral. D’un côté, on trouve la position de Detœuf pour qui la concentration des entreprises et l'apparition de monopoles sont des phénomènes intrinsèques à la logique du libéralisme historique. D’un autre côté, il y a Mises qui, déplaçant l’argumentation, soutient que seule l'action exogène de l'État a favorisé la création de cartels et de monopoles.

La position de Röpke reste ambiguë à ce sujet. Tout d'abord, il souhaite se démarquer fortement du libéralisme historique ${ }^{7}$ et pense que l'essor d'un renouveau libéral passe nécessairement par la prise de conscience des méprises passées (contrairement à Mises ou Hayek) :

6 Walter Lippmann (1889-1974) est un essayiste et journaliste américain. Son ouvrage The Good Society (1937) - traduit immédiatement en français sous le titre La Cité Libre (1946) - a été l'élément déclencheur d'activités transnationales entre intellectuels libéraux afin de renforcer et promouvoir leurs idées. Pour plus de détails sur le contenu de l'ouvrage et les positions de l'auteur, voir Clave, 2005. Neuf années avant la création de la Société du Mont-Pèlerin, le «Colloque Walter Lippmann» est bien souvent présenté comme un marqueur institutionnel de la naissance du mouvement néolibéral (Denord, 200 I ; Foucault, 2004a; Audier, 2008, p. 275-286).

7 Röpke entend par «libéralisme historique» la forme réelle qu'a pris l'économie de marché au $X I X^{e}$ siècle, se rapprochant pour lui du «Laissez faire», assimilé à la doctrine de l'école de Manchester et à l'utilitarisme. Ce libéralisme lui semble être un dévoiement de la doctrine initiale, par exemple telle qu'elle a été formulée par Tocqueville, Ferguson ou Smith. Röpke entend donc critiquer le «Laissez faire» sur les trois pans mis en relief par Jaffé: « I) le laissez faire comme fait objectif; 2) comme politique préconisée, et 3) comme hypothèse méthodologique d'économie déductive» (Handman et al. 1931, p.7, notre traduction). Du point de vue de l'histoire de la pensée, ses positions peuvent rétrospectivement paraîtres caricaturales; mais en la matière, elles rejoignent une lecture contemporaine partagée par plusieurs grands noms de la discipline à l'exemple d'Irving Fisher (1907) dans son Why has the doctrine of laissez faire been abandonned? ou de John Maynard Keynes dans son article sur La fin du Laissez faire (Keynes, 1999 [1926]). 
Il n'est pas possible d'ignorer que la ruine de l'ordre mondial, libéral et capitaliste, est due pour une part importante, à ses propres imperfections, à ses développements erronés et à ses dégénérescences. [...] Mais ce sont précisément des facteurs économiques et sociaux qui ont contribué à ce développement - et pour le traitement duquel nous devons avoir nos coudées franches, sans être liés dogmatiquement au programme économique du libéralisme historique (1945 [1942], p. 190).

Au regard de cette citation, il semble manifeste que les causes sont endogènes. Mais il n’en demeure pas moins que Röpke évoque parfois - et dans les mêmes ouvrages - le rôle prépondérant de l'État ${ }^{8}$ dans la concentration des entreprises (1936a, p. 8 ; 1945 [1942], p. 302 ; 1946 [1944], p. 272).

Quant aux causes économiques de la crise générale, Röpke attribue le déséquilibre social qui règne en Occident dans la première moitié du $\mathrm{xx}^{\mathrm{e}}$ siècle à un phénomène général qu'il nomme massification (Vermassung) de la société 9 . Sur le plan économique, la massification prend la forme d'une prolétarisation, entendue comme «une situation sociale et anthropologique caractérisée par une dépendance économique et sociale, le déracinement, l'encasernement, l'éloignement de la nature et l'anonymat du travail» (I945 [1942], p. 30-3I). Ce qui n'apparaît pas directement dans cet extrait, et qui pourtant en est le facteur séminal, c’est l’absence de propriété privée (1940 [1937], p. 289; I945 [I942], p. 232) ; d’où le fait - décisif chez Röpke - que ce trait culturel ne touche ni les paysans, ni les artisans.

La condition prolétaire découle directement de deux phénomènes consubstantiels au libéralisme historique: la concentration croissante des entreprises couplée à la division du travail et à la mécanisation des tâches. Après avoir discuté ces deux tendances, nous montrerons pourquoi elles représentent, chez Röpke, des conditions favorables à l'émergence du collectivisme.

$8 \mathrm{Si}$, au moins depuis la macroéconomie keynésienne, il est communément admis de considérer l'État comme un acteur manifestement endogène du circuit économique, on ne saurait soutenir un tel propos chez les théoriciens libéraux d'alors.

9 Ce thème, récurrent dans les principaux ouvrages de Röpke, fait écho au livre du philosophe espagnol José Ortega y Gasset, La Révolte des masses (1929) - disponible en anglais et allemand dès 1932 (Wuillème, 20II) - dont il partage de nombreux points de vue. 
Au début des années 1940, Röpke affirme que l'un des traits fondamentaux du capitalisme de ces cinquante dernières années c'est le nombre croissant de grandes entreprises, de cartels et monopoles. La concentration dans le domaine économique est de deux sortes, et toutes deux posent problème à leur façon. Il y a d'abord la «centralisation juridique et financière » (1946 [1944], p. 272) de différentes exploitations sous l'autorité d’un même mandant, que ce soit une personne physique ou juridique. C'est là la question du monopole. Il y a ensuite la «centralisation technique intérieure d'une unité d'exploitation» (ibid., p. 273), soit la question de la taille de la firme.

Röpke mêle ici réflexion économique et considération sociologique sur cette problématique de la concentration. En effet, sur le plan économique, la question primordiale est l'accroissement des monopoles qui anéantissent la concurrence de marché. Ce type de concentration ne suit pas une logique d'efficacité, mais elle est le fait de mobiles psychologiques irrationnels internes (1942, p. 273; 1945 [1942], p. 219) et/ou d'une ingérence politique externe. Cette dernière passe soit directement par la mise en place artificielle de certains monopoles, soit indirectement par une législation fautive: les tarifs, quotas et lois anti-faillites (1942, p. 226) ou relativement aux lois sur les brevets ${ }^{10}$, cartels et sociétés anonymes (1945 [1942], p. I57). À l'inverse, la concentration technique suit bien une logique d'efficacité (capital et progrès technique), même si Röpke note que l'on est surement allé trop loin, et au-dessus de la taille optimale, d'ailleurs pour les mêmes raisons psychologiques ${ }^{11}$. Mais l'argument réellement en sa défaveur est surtout d’ordre sociologique, car même si nous pouvions tout réaliser dans une seule et immense entreprise à des coûts minimes, le dommage social serait rédhibitoire (1942, p. 228):

I0 Röpke souhaite remplacer les brevets (qui établissent de fait des monopoles) par des licences obligatoires, soit des indemnités dues à l'inventeur (1945 [1942], p. 303).

II Par exemple, ceci explique pourquoi un «investissement de l'épargne de l'entreprise pour autofinancer ses installations au delà de la limite fixée par le taux d'intérêt qui aurait été payé si ce même capital devait être levé sur le marché» (1942, p.229) demeure répandu. 
N'est-ce pas la grande usine qui a tout transformé, l'homme, sa façon de penser, sa manière de vivre, son travail, sa foi, qui nous a dotés des déserts de pierres que sont les grandes villes avec leurs nomades, qui a affaibli tant de choses solides, rendu malade tant de choses essentielles, privé d'âme tant de choses vivantes? (1947, p. 29).

Certes, la division technique du travail et le machinisme sont des phénomènes concomitants à la concentration qui ont pris de l'ampleur, et qui vont, sur le long terme, engendrer des maux tant sur le plan économique que social. Le constat de Röpke est cependant plus nuancé : la division du travail est un phénomène indispensable de l'ordre économique libéral et le progrès technique a significativement amélioré les conditions matérielles de l'existence. D’une certaine façon, ces deux tendances sont liées et tiennent lieu de garde-fou à la concentration: «le développement récent de la technique des machines $[\ldots]$ a, de nombreuses façons différentes, exercé une influence à la baisse sur la taille optimale » (1936a, p. 7). Et pour Röpke cette tendance à la «spécialisation croissante » des petites entreprises serait «la plus profonde » cause de «progrès industriel» (1946 [1944], p. 277).

Il reste que Röpke insiste sur l'importance de conserver une structure économique qui satisfasse aux exigences de l'Homme, principalement par le sens de la mesure et de l'équilibre ${ }^{\mathrm{i} 2}$. Son réquisitoire relatif à l’abrutissement des corps et des esprits ouvriers (1940 [1937], p. II I et p. I3I) ne dépasse pas le constat exposé par Smith et s'accorde sur ce point avec Marx (Bonefeld, 20I3, p. I Io). Röpke exprime une indiscutable méfiance vis-à-vis du progrès technique, mais plus encore à l'endroit du machinisme, intrinsèquement néfaste ${ }^{13}$. Acquis aux thèses les plus conservatrices, il faut, à son sens, «opérer un certain retour au passé et augmenter le plus possible le secteur de l'auto-approvisionnement et des relations locales toutes simples de l’artisanat» (1945 [1942], p. I76). Outre ses effets sociaux, la division

12 «Le marché, la division du travail, la concurrence, le rationalisme économique ont ceci en commun qu'il y a dans leur emploi une limite à partir de laquelle les désavantages croissent plus vite que les avantages.» (1945 [1942], p. 158).

13 «Le problème de la machine [...] n'est pas une question de bon ou de mauvais emploi, mais procède de la nature même du machinisme, exigeant de l'homme un tribut parce qu'il suit des lois spécifiques et les lui impose.» (1945 [1942], p.66). 
technique du travail est un danger pour le système économique puisque croît avec elle l'instabilité de l'équilibre économique (1936a, p. 7I ; I940 [1937], p. I2I). L'expansion ou le basculement de l'économie dépend prioritairement du «règne de la sécurité monétaire et juridique» (1940 [1937], p. 92) qui est à même de contrebalancer les effets déstabilisateurs de la division technique du travail. «Une division du travail d’ampleur considérable ne peut donc se développer que dans la mesure où ces conditions - système monétaire, système juridique, système moral approprié - sont remplies» (ibid. ; voir aussi 1942, p. 72) et dont l'État doit être le garant.

Ces deux tendances que nous venons de décrire sont le résultat du libéralisme historique. Elles représentent, pour Röpke, les bases pré-collectivistes menant à l’audience contemporaine accordée au socialisme, ce qui l'autorise à dire que «le collectivisme est exactement le contraire de l'utopique, [...] il se réalise même si nous n'y prenons aucune part » (1940 [1937], p. 24) puisqu'il est le produit d'une dégénérescence interne. Finalement, il ne fait que se nourrir d'une tendance qui lui est profitable: «Le socialisme de tout crin n'offre qu'un changement de ce prolétarisme à double effet : il s'en nourrit pour créer l'idéologie correspondante et en même temps il le pousse jusqu'à ses limites. » En sorte que «le socialisme n’est pas autre chose que la conséquence logique d'une évolution engendrée et développée par un capitalisme dégénéré.» (1945 [1942], p. 32-33).

La croissance des monopoles et cartels, ainsi que la croissance de la taille des firmes et des coûts d'organisation sont les deux facteurs primordiaux à observer si l'on étudie la dérive collectiviste d'une économie nationale (I942, p. 224-225). Röpke note que lorsque des entreprises sont très (ou trop) grandes, elles deviennent en quelque sorte des entreprises nationales que l'État ne peut s'empêcher d'orienter, de soutenir et même de venir aider par la socialisation des pertes si nécessaires. En définitive, Röpke constate que la centralisation, la mécanisation et le morcellement des communautés au profit de groupes d'intérêts - rendus possibles par la philosophie du «Laissez faire» - ont ouvert la voie au collectivisme qui se nourrit d'une société de masse prolétarienne, laquelle sape les individualités au profit d'une dépendance au groupe qui, en plus de supprimer des libertés, offre un rendement matériel sous-optimal (1945 [1942], p. 229-230). 


\subsection{LES CAUSES SOCIO-POLITIQUES \\ Démographie et règles internationales}

Si la prolétarisation est l'expression économique de la massification, son pendant social et politique est désigné par le terme de grégarisme. Pour Röpke, le grégarisme consiste en «la simple agrégation des individus réduits désormais à eux-mêmes »; il ajoute plus loin : «c'est l'aplanissement de la pyramide sociale, son atomisation et, marchant de concert avec elle, la conglomération des individus» (1946 [1944], p. 221-222). Ce phénomène se manifeste de façon très diverse et totale, à la fois physiquement et psychiquement. Matériellement, c'est la concentration toujours plus importante des individus dans les centres urbains avec une désertification des campagnes. Au niveau intellectuel, c'est la culture et les idées de masse, l'abandon des communautés naturelles - que sont la famille, la commune, l’Église et la profession (1945 [1942], p. 25) - ou des hiérarchies et le repli individualiste. C’est en somme, dans tous les domaines, célébrer le goût du colossal. C'est selon lui un trait fondamental du collectivisme d'inspiration marxiste comme national-socialiste :

Le culte du colossal, c'est tout d'abord fléchir le genou devant la simple «grandeur», admise comme une preuve suffisante de qualité et de valeur; c'est mépriser la petitesse, même si elle renferme la grandeur; adorer la puissance et l'unité; cultiver le superlatif dans tous les domaines de la vie culturelle (ibid., p. 94).

La principale cause de ce phénomène de massification est exogène, c'est l'explosion démographique (1936a, p.6; 1940 [1937], p. I06-107; 1942, p. 24I). Mécaniquement, l'augmentation numérique de la population dans l'Occident du XIX ${ }^{\mathrm{e}}$ siècle a rendu impossible le processus normal d'assimilation spirituelle des individus, altérant par la même les structures sociales et économiques par une concentration toujours croissante. Étonnement, la cause essentielle est donc matérielle et non d'ordre spirituel. Implicitement, Röpke pondère cette incohérence formelle lorsqu'il met en évidence à quel point la philosophie rationaliste et l'essor d'une mentalité de masse sont deux phénomènes inextricablement apparentés ${ }^{14}$ (1945 [1942], p. 94).

I4 II s'inquiète de la croissance démographique sans jamais témoigner la moindre sympathie - ou antipathie - envers les thèses eugénistes du début du siècle. 
Outre la démographie, cette société de masse est entretenue par différents vecteurs: I) hausse de l'individualisme ${ }^{15}$ et 2) baisse de la satisfaction vitale apportée par les conditions urbaines de travail - dans le domaine moral; 3) évolution technologique des grandes entreprises et 4) politiques sociales et institutionnelles fautives - pour les phénomènes d’ordre réel.

La société de masse, pour Röpke, ne touche pas nécessairement une majorité de la population, c'est plus un état d'esprit significatif des groupes dominants : la «bourgeoisie décadente» (ibid., p. 23). Aussi, le terme de «masse» renvoie moins à certaines classes sociales ou catégories socio-professionnelles qu'à une mentalité spécifique pouvant frapper n'importe quel individu sur l'échelle sociale:

Il serait faux et présomptueux d'assimiler ce terme de «masse» aux couches populaires à petits revenus ou de peu de propriété. Bien au contraire, il s’agit d'un processus de dégénérescence indépendant de l'échelle des revenus, et dont certaines classes peu aisées sont encore les moins atteintes, par exemple celle des paysans et des artisans (ibid., p. 29).

Röpke s'inquiète de l'absence d'une «élite conductrice» (I940 [1937], p. 285), seule à même de sortir la société de cet état d'esprit. Si la définition du terme «masse» est claire, tel n'est pas le cas de ce que l'auteur entend par «élite». En effet, il nous assure préalablement que cette catégorie peut également être présente à tout niveau de la société, avec un biais favorable à la classe moyenne (1946 [1944], p. 20I). Mais force est de constater qu'il considère cette élite - constituée de clercs $^{16}$ - comme relevant de catégories professionnelles expressément indiquées : les scientifiques, la presse et la magistrature (ibid., p. 200-2I3).

En plus de causes sociales dues à la grégarisation, la crise contemporaine s'est également accomplie via des phénomènes plus explicites, et notamment par des décisions politiques au plan international. Les échanges entre nations ont été un formidable moteur de développement au XIX siècle, mais ils sont devenus,

15 Ce qui se manifeste par la perte de l'intégration sociale, l'atomisation, la diminution des différences de statuts et la hausse de la standardisation et de l'uniformisation qui détruisent la cohérence verticale de la société; ou encore «le remplacement progressif de l'ordre spontané et de la cohérence au profit de l'organisation et de l'embrigadement 》(1942, p.240, notre traduction)

16 Reprenant le terme du célèbre ouvrage du philosophe français Julien Benda, La Trahison des Clercs (1927). 
pendant la période d'entre-deux-guerres, un facteur d'instabilité qui a contaminé les économies nationales. Il apparaît clairement que le commerce international est plus fragile, de fait, puisqu'il ne peut que sporadiquement compter sur un cadre d'institutions et de lois communes ${ }^{17}$, contrairement à l'échelon national. Il souligne que par le passé, ce n’est pas grâce au libre échange exacerbé, mais justement à la prééminence de règles internationalement admises ${ }^{18}$ que le succès des échanges a été assuré. Celui-ci repose sur deux piliers: d'un côté l'étalon-or - représentant virtuellement une communauté de payement (1942, p. I6) - et de l’autre le traitement du crédit entre les nations. Röpke signale qu' «en dernier ressort, tous deux représentaient bien plus que des arrangements techniques: ce sont des institutions morales et elles ne peuvent subsister qu'en tant que tel» (ibid., p. 76, notre traduction, souligné par nous). L'effondrement de ce système dans les années 1920 a précipité le monde dans la crise (ibid., p. 77-78).

Il ne faut pas se méprendre, si le déclin frappe toutes les sphères de la société et que son impact est diffus sur l'ensemble de l'Occident, il existe de grandes disparités entre les pays. Dans son ouvrage Civitas Humana (1946 [1944]), Röpke propose une typologie d'États sains et d’États malades, reprenant en grande partie des constats déjà exposés dans ses précédents travaux. Le caractère sain / malade renvoie à trois caractéristiques : légitime / illégitime, libéral (solidariste) / autoritaire et décentraliste / centralisateur (ibid., p. I60). Les Pays-Bas, la Grande-Bretagne ou les pays nordiques sont considérés comme sains, par opposition à la France et l'Allemagne qui présentent néanmoins des défaillances distinctes : «Si nous comparons entre eux les différents pays, nous en retirons l'impression qu’en général les hommes ont seulement le choix entre l'ordre et la discipline autoritaire (ex. l'Allemagne) ou la liberté et le nonchalant laissez-faire (ex. la France et l'Autriche). (ibid., p. I63).

17 En effet, Röpke note: «bien que, dans sa substance économique, le commerce international ne soit pas essentiellement différent du commerce national, il est clair qu'il a toujours été handicapé par la grande fragilité et la précarité de sa base socio-politique» (1942, p. 72-73, notre traduction).

I8 Ainsi le rôle de l'Angleterre et de la Pax Britanica (1815-1870) qu'elle engendra est perçu très favorablement par Röpke qui considère comme extrêmement prégnant le rôle des traités, des règles implicites et d'une certaine moralité des échanges (1942, p.73-74). 
La France concentre de nombreuses critiques (Campbell, 2002 [1995], p.xviii) en raison de sa tradition rationaliste, révolutionnaire et hautement centralisatrice. À l'inverse, Röpke considère la Suisse comme la plus aboutie des démocraties libérales, mariant sens de la mesure et équilibre entre sur- et sous-intégration sociale ${ }^{19}$. Le pays des Helvètes est inlassablement cité à titre d’exemple (1942, p. 5 et p. 266; 1945 [1942], p. 47 et p. 233-234; 1946 [1944], p. 9 et p. 248), il constitue pour l'auteur l'«idéal traduit dans la plus concrète des réalités ( (1946 [1944], p. 70-7I). L’effet «taille» joue ici un rôle très important, à tel point que l'on peut se demander si le modèle parfait préconisé par Röpke est transposable à des pays de dimension plus importante.

\section{I.3. LES CAUSES PHILOSOPHIQUES ET MORALES Fides et Ratio}

Si cette dégénérescence dans le domaine économique, politique et social est de grande importance et mérite d'être étudiée spécifiquement (comme l'a d’ailleurs fait Röpke), elle ne représente finalement que la partie visible de l'iceberg. Aussi, ces deux premières parties ne font sens et ne s'interprètent quau regard de la critique philosophique et morale. Pour Röpke - idéaliste (au sens philosophique) convaincu $^{20}$ - le monde occidental vit sur un «un capital spirituel et moral que l'antiquité païenne et le christianisme [...] ont légué comme un héritage inaliénable» (1945 [1942], p. 76). Le monde contemporain reflète donc le cours du conflit des idées du XIX ${ }^{e}$ siècle. Bien qu'étant identifié comme une période, par de nombreux aspects, hautement libérale ${ }^{21}$ et donc féconde en idées plébiscitées

19 La sous-intégration sociale signale un manque de communauté, un individualisme trop marqué (c'est l'apanage du laissez-faire). A contrario, la sur-intégration sociale se traduit par trop d'arbitraire et de tyrannie collective (ce sont les conséquences néfastes du socialisme). La sur-intégration est un phénomène normal en temps de crise et de catastrophes; ce qui pousse Röpke à dire que le collectivisme est le système typique d'une économie de guerre et qu'il cherche toujours à alimenter les conflits pour survivre (1945 [1942], p. I23-124). Idéalement, l'auteur cherche une intégration médiane qui réponde à l'«insociable sociabilité» de I'Homme mise en évidence par Kant (1942, p. 257; 1945 [1942], p. 123).

20 «[...] la littérature est l'expression même de la société d'aujourd'hui et elle crée celle de demain; les idées sont les véritables souveraines du monde » (1945 [1942], p. 56).

21 Malgré les crises ou le retour à des mesures protectionnistes (notamment aux États-Unis et sous l'Allemagne de Bismarck, dès les années 1870). Par contraste, Röpke considère la période de 1815-1914 comme essentiellement libérale (1942, p. 18; 1959a, p. 23). 
par Röpke (1936a, p. II-I2), le monde contemporain est paradoxalement marqué par l'envol d'idéologies et de principes nocifs à l'égard de l'«essence même de l’homme» (1946 [1944],p. 252). Cet idéalisme doit être qualifié de chrétien tant l'auteur insiste sur les liens inextricables entre pensée chrétienne, humanisme et libéralisme. De confession protestante, il accueille avec bienveillance et intérêt les réformes sociales préconisées par l'Église catholique, notamment l'encyclique du pape Pie XI, Quadragesimo Anno de I93I (ibid., p. I2).

En ce sens, il s'oppose à $\mathrm{Marx}^{22}$ par deux fois : contre le matérialisme et l'athéisme. Il fait de ce dernier le fossoyeur des idées libérales, dans le sillage de Saint-Simon et de Rousseau (1987 [1951], p. IO). Il reproche également à l'auteur du Capital le déterminisme impliqué par des forces irrésistibles de nature objective et sa façon d'idéologiser l'économie, d'en faire le reflet d'une volonté d’impérialisme et de domination, soit une lutte des classes perpétuelle (1936a, p. I3 ; 1940 [1937], p. 59). Paradoxalement, il déplore l'approche exclusivement négative et critique du capitalisme faite par le courant marxiste, alors qu'il devrait se concentrer sur la structure possible d'une société socialiste sans attendre une réalisation prétendue inévitable (1936b, p. 319).

Si ses critiques à l'endroit de la pensée marxiste s'avèrent souvent grossières et sans originalité, il est intéressant de noter que pour Röpke, marxisme comme libéralisme historique se sont rendus coupable du même aveuglement et de la même «sociologie funeste de l'économicisme» (1945 [1942], p.48). En effet, et nous y reviendrons ultérieurement, ils se sont tous les deux trompés dans leur vision des intérêts économiques en tant que force sociologique motrice. Mais la critique du marxisme n'est pas le seul cheval de bataille de Wilhelm Röpke. Une philosophie plus profonde - qui englobe d'ailleurs le marxisme - est à l'œuvre : le «rationalisme technico-scientifique» (ibid., p. 203). Si Röpke croit en l’apport primordial de la raison, il s'inquiète de la montée en puissance du positivisme (I940 [1937], p. I7 ;

22 Les fondateurs de l'école de Fribourg ont également à cœur de se positionner explicitement en opposition au marxisme dans ce qui sera rétrospectivement qualifié de Manifeste Ordolibéral de 1936 (Böhm, Eucken et Grossman-Doerth, 1989 [1936]). Röpke se sent plus proche de socialistes français comme Proudhon et avoue partager les analyses de Sismondi (1945 [1942], p. 255). 
I945 [1942], p. I9). Dans son esprit, positivisme, matérialisme et socialisme vont de concert et représentent des errements du rationalisme ${ }^{23}$. Ils procèdent, au fond, d'une erreur identique. Tous méconnaissent ou nient carrément l'existence de certaines valeurs transcendantales inhérentes à la condition humaine en sociétée ${ }^{24}$ :

C'est une pensée qui passe, sans s'en douter, à côté de l'homme en tant qu'être normal et spirituel et qui ne sait pour ainsi dire rien de toutes ces valeurs éternellement sociales et humaines, de ces relations, de ces problèmes qui, pour la pensée, sont «inexacts», parce qu'ils doivent être pénétrés par une culture philosophique, littéraire, historique (1946 [1944], p. 108).

En cherchant l'inconditionnel et l'absolu, le quantitatif, le mathématique et le physique, le rationalisme s'est perdu : «une pareille mentalité rend aveugle sur les nécessités de la vie qui, elle, est qualité, structure et forme » (1945 [1942], p. 70). Pire, le positivisme a précipité l'essor du nihilisme en Allemagne qui, en plus de mener au relativisme des valeurs (1946 [1944], p. 109), a également engendré un recul de la confiance envers les travaux scientifiques (1945 [1942], p. 22).

Nous l'avons évoqué, libéralisme et socialisme sont coupables du même aveuglement économiciste «qui s'en tient à l'aspect économique et à la productivité matérielle; [...] fait du monde matériel et économique la pierre angulaire qui supporte tout l'édifice, rapporte tout à lui et le lui subordonne comme un simple moyen d'arriver au but » (ibid., p. 75). Concentrons-nous sur les reproches adressés au libéralisme historique puisqu’il représente la logique économique dominante du XIX siècle, dont les contradictions internes faciliteront l'apparition d'un mal, jugé plus grand encore ${ }^{25}$.

23 Sur ce sujet, le constat de Röpke rejoint celui de Friedrich Hayek (1941 et 1948; voir l'analyse de Lagueux, 1988) sans proposer exactement la même critique ni en tirer les mêmes enseignements, notamment quant au constructivisme et aux politiques «sociales». Röpke fera du keynésianisme, à l'instar de son collègue autrichien, l'expression contemporaine de ce rationalisme exacerbé (1956, p. 120-121).

24 Par exemple, le socialisme tombe dans ce piège lorsqu'il «construit son État idéal sans tenir compte de la nature immuable de l'homme et du caractère vital et anthropologique de la propriété» (1945 [1942], p.69, souligné par nous).

25 «Une seule différence avec le collectivisme, c'est que la philosophie du laissez-faire ne nous inquiète point comme le collectivisme par sa présence actuelle, mais uniquement par son fatal héritage, I'héritage du monde d'aujourd'hui que ce libéralisme historique constitua avec autant d'aveuglement que de zèle.» (1946 [1944], p.99-100). 
Si le libéralisme historique a échoué et mené à sa propre disparition ainsi qu’aux effets néfastes du début du $\mathrm{xx}^{\mathrm{e}}$ siècle, c'est parce qu'il a profondément ignoré toutes les conditions sous-jacentes à l'essor d'une économie de marché libérale. Ces conditions avaient bien été identifiées par les pionniers de la discipline $^{26}$, mais, par la suite, les tenants du libéralisme ont abandonné ces considérations pour se concentrer exclusivement sur la grande découverte de l'économie politique : «le marché réglé automatiquement par la concurrence » (ibid., p. 72). Or, toute l'argumentation de Röpke consiste à dire que l'un ne va pas sans l'autre et que pour assurer l'autorégulation du marché il faut se préoccuper des conditions qui l'instituent et la rendent possible. Le libéralisme historique méconnaît les conditions politiques, sociologiques et morales au profit d’un «ordre naturel» et de la seule «main invisible» (1946 [1944], p. I05).

Si le mécanisme concurrentiel est le principe interne essentiel qui permet l'autorégulation, comme le soutient Röpke (1945 [1942], p. I69-I70), il n’est pas pour autant un principe adéquat à la régulation de l'ensemble du corps social ; de sorte que le problème se pose sur deux plans. Ex ante, il faut assurer l'ensemble des conditions fondamentales à l'accomplissement des effets positifs de l'économie de marché. Mais ex post, les effets sociaux de la concurrence peuvent être désastreux pour la communauté :

Que la concurrence fût un danger moral et sociologique à limiter et à surveiller afin de prévenir tout empoisonnement du corps social, voilà une vérité qui reste cachée à ce libéralisme historique et surtout à celui du XIXe [ ...] Aujourd'hui, nous savons que l'économie fondée sur le libre jeu de la concurrence suppose de fortes réserves morales à prélever en dehors de l'économie du marché, car elle en consomme au lieu de les alimenter (ibid., p. 72-73).

26 «Nous avons beaucoup à apprendre du XVIII siècle, et, en tant qu'économistes, nous ne devons pas nous soucier exclusivement des problèmes économiques, mais aussi, dans la mesure de nos forces et de nos connaissances, des fondements juridiques, sociologiques, anthropologiques, politiques, moraux et même théologiques de la société. » (1940 [1937], p. 17). 
Les économistes de tradition classique ont eu tort de considérer le marché concurrentiel à la fois comme sociologiquement autonome - celui-ci dépend de son cadre et, en retour, l'influence et le modifie (1942, p. 68) -, et comme vecteur d'effets sociaux majoritairement positifs. Le libéralisme historique a été, pour Röpke, victime du rationalisme et du naturalisme, considérant admise l'harmonie naturelle des intérêts, qu'il ramène fondamentalement au darwinisme social (1945 [1942], p. 212). Rien n'est moins naturel que cette harmonie et la réaliser consiste non pas en un travail négatif d’abolition des obstacles (1946 [1944], p. 99-100), mais en un travail positif sur ses fondements hors de la sphère économique et devant lui préexister. La dernière méprise du libéralisme historique consiste en sa conception de l'individu :

L’aveuglement sociologique avec lequel on plaçait à la base de l'économie l'individu libre dans l'univers, tel un atome sans aucune attache, correspondait aussi à l'exagération rationaliste du principe de la concurrence, reposant sur l'égoïsme individuel. Par là même, on ressentait nécessairement comme des entraves gênantes les forces et les liens obligatoires que sont la famille et les communautés naturelles du voisinage, de la commune, de la profession, etc. Il en résulta cet individualisme funeste, dont on a enfin reconnu le caractère destructeur pour la société, et qui a réussi à discréditer une idée juste au point de favoriser le progrès du collectivisme, infiniment plus dangereux encore. Il n'est donc point étonnant que ce libéralisme économique, issu du rationalisme, se soit mis à ignorer souverainement les données vitales et anthropologiques capables de circonscrire les empiètements de l'industrialisme capitaliste, et qu'il ait volontairement imposé aux hommes une manière de vivre contraire à leur nature et contre laquelle ils se révoltent pour finir (1945 [1942], p. 74).

On touche ici aux véritables causes de la débâcle libérale: les idées rationalistes, naturalistes et individualistes ont conduit à un cadre (a-)moral qui a sapé les fondements nécessaires au bon fonctionnement de l'économie de marché. Les maux sociaux n'ont fait que renforcer cette crise des valeurs, produisant de l’anomie sociale. Pour autant, Röpke ne condamne pas le libéralisme en général, faisant de cette manifestation particulière l'expression erronée d'une essence profondément salutaire. Mais son libéralisme n'est pas un universalisme puisqu'il reste profondément attaché à la contingence historique de la culture européenne. Il cherche, au fond, à passer d'un libéralisme historique à un libéralisme historiscite. 
Son adhésion aux principes du libéralisme se situe bien plus sur le plan moral que technique ou pratique (1959b, p. 232 ; 1964, p. 196). Malgré les effets socialement et moralement néfastes, il reconnait l'incroyable production de richesse dégagée au XIX siècle; il n'empêche qu'à son sens les bienfaits matériels ne compensent pas cette déconvenue. Ainsi, de présupposés philosophiques du libéralisme historique découle une certaine vision de la société et de l'économie oublieuse des conditions sociologiques et institutionnelles de base, et produisant des effets socio-économiques qui peuvent être ramenés, de manière générale, à une crise de la société des «masses» (1940 [1937], p. I8; 1942, p. 239).

Sans qu'il s'agisse de mettre en cause directement le fondement des analyses de Röpke, l'on remarquera que son diagnostic pose problème à deux niveaux au moins. Tout d'abord les enchaînements causaux et les mécanismes de propagation d'une sphère à l'autre ne sont pas détaillés ou se révèlent généralement confus. Ce sont des faisceaux de causes et d’effets souvent circulaires. L'argument d'interdépendance générale de toutes les sphères de la société invoqué par l'auteur ne modère que trop imparfaitement cette faiblesse dans l'analyse. Par souci de réalisme, ce postulat lâche favorise l'irréfutabilité de la lecture historique de Röpke. En effet, il permet de rejeter toute anomalie d'une analyse circonscrite à un domaine - par exemple économique - à une influence externe qui provient d'une autre sphère, et/ou d'une autre époque. Enfin, le passage de l'ensemble des conditions morales, sociopolitiques et économiques du déclin à la situation de crises effectives, comme en 1929, est absent des considérations de l'auteur. Pour autant, il est à notre sens fondamental de comprendre le diagnostic établi par Röpke, car il représente la base heuristique nécessaire à l'élaboration de son libéralisme conservateur. 


\section{RÉPONDRE À LA CRISE SOCIALE Les tensions du libéralisme röpkéen}

Dans cette seconde partie, nous tâcherons de reconstruire en miroir ce libéralisme röpkéen en tant que réponse aux trois grandes catégories de causes précédemment exposées: une vision de la science qui vient pondérer le rationalisme scientiste; les bases organisationnelles d'une démocratie à même de combattre le grégarisme ; enfin, un interventionnisme libéral actif face à la concentration économique.

\section{I. MORALE ET SCIENCE ÉCONOMIQUE Retour à l'économie politique}

Wilhelm Röpke ne souhaite privilégier aucun type de recherche scientifique plutôt qu'un autre a priori. Il juge néanmoins que l'un des problèmes de la science économique ${ }^{27}$ contemporaine tient dans une vision réduite de la classe des phénomènes «économiques » laissant de côté les questions sociétales dans leur ensemble (1942, p. 2). Et selon les différentes phases qu’il étudie, il se réfère à des travaux tantôt très spécialisés tantôt plus généralistes, ou pluridisciplinaires. En effet, Röpke distingue les périodes de prospérité sociale des périodes de crise générale, auxquelles chaque type de recherche apporte des réponses adaptées selon les différents besoins sociaux qui s'expriment.

La limitation et les travaux de détail spécialisés étaient utiles et même, historiquement, nécessaires aussi longtemps que la société, prise dans son ensemble, était à peu près intacte. Mais cette période idyllique de la spécialisation a perdu aujourd'hui son fondement, attendu que les données d'où elle procédait sont devenues caduques et que nous avons désormais affaire à une crise sociale très grave et totale. Maintenant, la société doit de nouveau être considérée comme un ensemble, ensemble qu'elle constitue en réalité (1946 [1944], p. 17).

27 Pour Röpke, «faire de l'économie n'est rien d'autre que le choix incessant entre différentes possibilités, et l'économie politique n'est au fond que la science de l'alternatif» (1940 [1937], p.46) ce qui semble directement inspiré de la définition, aujourd'hui canonique, proposée par Lionel Robbins dès 1932: «science qui étudie le comportement humain en tant que relation entre les fins et les moyens rares à usages alternatifs » (Robbins, 1947, p. 30). 
Au cours d'une période de prospérité, une recherche spécialisée est féconde dans la mesure où la stabilité des autres domaines de la vie sociale permet de travailler comme s'ils étaient fixes, en faisant abstraction de la réalité d'une structure générale. Mais dans les périodes de troubles, les changements sont incessants et le besoin d'harmonie supplante le besoin de spécialisation.

Par ailleurs, son appel à des travaux d'ensemble porte sur un effort de synthèse plus que sur de nouvelles analyses (1936a, p.vi; 1940 [1937], p. I6; I946 [1944], p. I44) : «mettre de nouvelles doctrines à la place des anciennes n'est ni un besoin, ni une nécessité » (1940 [1937], p. I5). Il semble très bien se satisfaire de la doctrine classique augmentée des apports du marginalisme autrichien. Röpke les perçoit, si ce n'est comme proprement compatibles, tout du moins comme complémentaires (ibid., p. 5I).

Röpke est fermement attaché à une méthode théorique et hypothético-déductive (1956, p. I16-II7), couplée à une approche morphologique (1942, p. III) héritée de la tradition historiciste allemande ${ }^{28}$. Il reconnaît également l'avancée et l'intérêt indéniable de l'analyse empirique basée sur une formidable collecte de données et la recherche de relations causales (1936a, p. 65). Mais ce travail vient dans second temps pour confirmer ou infirmer le modèle théorique, seul à même de faire réellement progresser la recherche. Chez Röpke, l'économie est avant tout une science morale, mais, par les phénomènes particuliers qu'elle entend analyser, elle doit être capable de se munir d'outils qui sont étrangers à cette tradition, car elle est une «véritable "science de frontière" » (1956, p. I22, notre traduction).

L'économie traite bien des quantités, mais Röpke perçoit comme une dérive le recours croissant et l'attractivité exercée par l'économie mathématique. Il y voit un moyen de passer outre toute considération morale ainsi qu’un traitement mécanique du corps social, s’appuyant sur des agrégats fautifs. Il

28 Moins explicite sur son épistémologie que ne le fut Walter Eucken dans son maître ouvrage (1950 [1940]), Röpke semble partager une méthodologie relativement similaire. II évoque l'œuvre de son confrère fribourgeois en des termes élogieux et, par exemple, fait sienne la typologie des formes d'organisation de l'économie qu'il propose (1946 [1944], p.19, p. 32 et p.78 [n.3]). L'épistémologie de Walter Eucken peut être vue comme une tentative de synthèse visant à dépasser la querelle des méthodes allemandes (Broyer, 2007). 
rejoint les positions autrichiennes en la matière (Leen, 2003). L'usage doit être clairement instrumental et modéré :

L'économie peut, dans les faits, permettre un recours aux mathématiques pour illustrer ou formuler avec précision des relations causales de caractère quantitatif. Et il y a en effet peu d'économistes modernes qui chercheront à rejeter toute forme d'usage des mathématiques. Mais cette méthode-même est ouverte à discussion parce qu'elle attire les imprudents à repousser exagérément la frontière qui délimite le bord du territoire, la zone entre ce qui est humain et mécanique (1956, p. I22, notre traduction).

Derrière ces considérations d'ordre épistémologique (qui découlent encore, en dernier ressort, de ses conceptions morales), il ne faut pas oublier qu'on trouve également une prise de position théorique et politique à l'encontre de la possibilité d'un «calcul socialiste $»^{29}$, et plus tard une opposition à la macroéconomie keynésienne.

Afin de se prémunir de toute dérive positiviste et de réintégrer des postulats qu'il juge fondamentaux, Röpke souhaite réhabiliter dans le domaine de la science économique les jugements de valeur. Doivent ainsi être comprises dans le domaine scientifique certaines valeurs suprêmes ainsi que les positions ou critiques relatives à ces mêmes valeurs. $A$ contrario, l'ensemble des velléités politiques, des idéologies ou des raisonnements partisans relèvent de la nonscience. Le critère que Röpke identifie pour discerner dans laquelle des deux sous-catégories doivent être placées certaines affirmations repose sur l'universalité de leur acceptation : à savoir, lorsqu'elles «revêtiront un caractère carrément objectif, [...] assuré de l’assentiment général» (1946 [1944], p. I39). Cette frontière semble superficielle, poreuse, voire peu rigoureuse; elle concerne finalement une minorité de principes élémentaires comme «la vérité, la justice, la paix, la communauté » (Wörsdörfer, 20I2, p. I I, notre traduction) :

29 Initié dans les années 1920 par Mises, le débat a connu différentes étapes et malgré son essoufflement suite à la chute du bloc communiste, il connaît une postérité relativement importante. Comme l'indiquent Levy et Peart (2008), Eucken (1948) apporte une contribution importante au débat. L'apport analytique de Röpke, en la matière, est insignifiant puisqu'il se positionnera plus volontiers sur le plan politique et moral. 
Ils ne sont rien de moins que des faits anthropologiques que la science doit respecter aussi bien que d'autres faits, et sur lesquels elle a le droit incontestable, et même le devoir, d'opérer ainsi qu'elle fait pour tous les autres faits objectivement donnés. [...] Exactement comme pour les mots, il en est de même pour toute idée élémentaire de norme et de valeur concernant la vie de la société et de l'économie (I946 [i944], p. I4I).

Röpke ne cherche pas à opérer une scission entre science positive et normative, il discerne plutôt une source de renforcement mutuel qui n'affaiblit pas le caractère scientifique de l'économie. Aussi a-t-il été souligné que cette distinction doit être considérée «comme un continuum qui implique des énoncés d'une haute valeur positive à l'une des extrémités du spectre, et des revendications éminemment normatives de l'autre» (Gregg, 20I0, p. 69, notre traduction).

Le renouveau de la science et des scientifiques est primordial, au-delà du fait que l'économie doit guider les politiques publiques, elle a un autre rôle tout aussi important : éduquer et renseigner l'individu sur la place qu'il occupe dans le processus. Pour que le système fonctionne, il faut que les individus comprennent ses rouages, ses lois et sa structure. La voix des scientifiques doit éclairer le débat politique en éliminant la démagogie et la superstition économique: «L'économie devrait être la science par excellence qui désillusionne, qui est antivisionnaire, anti-Utopique et anti-idéologique.» (1956, p. I27, notre traduction).

Cette science doit être le fait d'une classe de clercs à même de guider l'évolution de la société. Ce faisant, elle encourage et délimite l'action publique permettant de rapprocher le monde actuel du monde rationnel, guidée à l'aide de principes façonnant une politique constructive (I942, p. 233).

\section{2.ÉLITISME, ANTI-PLURALISME ET DÉMOCRATIE ÉCONOMIQUE}

L'interdépendance entre les sphères morale, politique et économique est un phénomène auquel l'auteur accorde la plus haute importance, comme nous l'avons vu dans la première partie de ce travail. Mais à côté de la nécessité de penser cette interdépendance, il s’avère également primordial d’assurer une parfaite étanchéité entre ces sphères. Pour le dire autrement : l'essence même d’une société libérale et démocratique, chez Röpke, c’est précisément cette démarcation entre politique et 
économique ${ }^{30}$. Pour autant, cela n’autorise pas à promouvoir des logiques complètement différentes d'une sphère à l'autre, du fait précisément des interdépendances :

Il existe toujours entre la politique et l'économie une corrélation qui interdit de combiner n'importe quel système politique avec un système économique et vice versa. [...] La dictature économique ne peut à la longue exclure la tyrannie politique et spirituelle et, inversement, la dictature politique et spirituelle engendre fatalement la tyrannie économique. Croire qu'un État peut être «total» dans le domaine économique sans l'être également sur le plan politique et spirituel, et vice versa, serait bien naïf (1945 [1942], p. I18, p. I22 ; voir aussi 1946 [1944], p. 54).

C'est formellement la fonction de l'État que de «conjuguer ordre et liberté » (1946 [1944], p. I53). Il faut entendre «ordre» de deux façons complémentaires: comme principe d'autorité, de hiérarchie et comme forme d'ordonnancement. Il n'y a pas de frontière établie entre le domaine politique (ordre) et le domaine du marché (liberté), mais plutôt un équilibre qui appelle des principes d’ordre et de liberté au sein de chacune des sphères :

[...] la démocratie - plus souvent encore que d’autres formes d'État - peut conduire au pire despotisme et à l'intolérance si elle n'est pas limitée par d'autres principes et d'autres institutions. Ces limites, dans leur ensemble, sont précisément ce qu'il est convenu d'appeler le contenu libéral d'une structure politique (1945 [1942], p. I14).

Le principe de marché suppose, lui aussi, certaines limites et si la démocratie doit avoir des sphères soustraites à l'influence de l'État pour qu'elle ne tourne pas au despotisme outrancier, l'économie de marché doit en avoir également qui ne soient pas soumises aux lois de marché, sous peine de devenir intolérables: nous entendons la sphère de la communauté et du dévouement pur, la sphère de l'auto-approvisionnement, la sphère des conditions de vie toutes simples et modestes, la sphère de l'État et de l'économie planifiée (ibid., p. 159).

Dans cet esprit, «ordre » et «liberté » n’ont de sens et d'existence réelle que par complémentarité. Röpke a toujours en tête cet équilibre lorsqu'il propose de nouvelles bases organisationnelles ou une délimitation du champ des interventions de l'État, s'inscrivant de plain-pied dans la tradition de l'économie

30 Symétriquement, l'auteur fait remarquer que l'économie fasciste fait passer le politique avant l'économique, soutenu par un État corporatiste (1935, p. 95). Il partage le même point de vue sur le socialisme: «la politique envahissant l'économie toute entière définit parfaitement le socialisme» (1945 [1942], p. 128). 
politique comme réflexion sur le bon gouvernement (Foucault, 2004a; 2004b). Nous allons maintenant aborder plus en détail ses propositions dans la sphère politique, celles dans le domaine économique faisant l'objet d'une partie finale.

Afin de lutter contre le grégarisme, plusieurs types de réformes sont requises : à la fois directement sur l'action gouvernementale, mais aussi sur l'organisation générale de la structure de l'État. Le gouvernement doit être exercé par un État «fort», dans un sens premier ${ }^{31}$ d'incorruptibilité et d'imperméabilité totale aux pressions potentielles des groupes d'intérêt: c'est l'anti-pluralisme d'un État impartial (Megay, 1970). Il est ainsi étranger aux revendications dans le domaine de la lutte des classes, de la haine raciale et du nationalisme ou encore des conflits religieux (1946 [1944], p. 165) qui sont généralement l'expression de pulsions des masses.

La démocratie idéale de Röpke renvoie à une inaccessible «unanimité réelle de la volonté populaire » dont il faut se rapprocher en assurant certaines conditions comme le sens de la communauté (pensées et réactions identiques), une décentralisation poussée, ou encore un «gouvernement [qui] limite son activité aux seules tâches pour lesquelles il peut tabler sur un acquiescement général qui ne s'étende pas au-delà du cadre de ses fonctions gouvernementales légitimes parce qu'elles appartiennent à l'essence même de l'État » (1945 [1942], p. I20). Politiquement, l'État doit accepter une constitution politique et économique inviolable qui sacralise quelques principes essentiels acceptés de tous, lesquels vont orienter le champ de ses actions valides (renvoyant à une forme de contrat social).

31 Cette vision de l'État «fort» (Jackson, 20I0; Bonefeld, 20I2) ne doit pas être confondue avec l'État «total» de Carl Schmidt, malgré l'influence certaine qu'a exercé cette doctrine de philosophie politique sur Röpke - notamment par le biais de Rüstow (Ptak, 2009) - et bon nombre d'auteurs ordolibéraux comme Walter Eucken ou Franz Böhm (Commun, 2009). Röpke évoque succinctement la pertinence de certaines analyses de Schmidt tout en reconnaissant leurs limites, il les juge, en définitive, antilibérales (1959a, p. 208). En effet, l'une des différences centrales c'est le glissement opéré d'une logique d'exercice du pouvoir par le biais de l'exécutif (Schmidt) à une logique au profit du législatif (ordolibéralisme). Röpke s'insère parfaitement dans cette dernière catégorie puisque légiférer représente, à son avis, la meilleur expression de l'autorité de l'État (1945 [1942], p. 253). 
L'anti-pluralisme passe par le rejet catégorique d'une démocratie représentative parlementaire et proportionnelle; le libéralisme röpkéen préfère une voie plus élitiste. Cet élitisme s'incarne non seulement dans une certaine méfiance vis-à-vis de la capacité des masses à choisir les meilleures solutions et à s'exprimer en leur faveur, mais également dans leur capacité à élire les bons représen$\operatorname{tants}^{32}$. Il pousse Röpke à encourager un corps politique dont les fonctions seront de plus en plus déléguées à des experts indépendants des logiques de nomination populaires ou de la rigidité bureaucratique. D'où l'extrême confiance qu'il accorde aux juges (ibid., p. 253), une des trois grandes composantes des clercs.

Le pendant de cet élitisme est la volonté de renseigner les masses et d'instruire le peuple en vue d'une meilleure connaissance des mécanismes de l'économie de marché libérale. À ce titre, «rien n’est plus important que la clarification la plus complète de tous les phénomènes économiques, la publicité la plus large et la plus transparente, l'examen le plus parfait possible des données qui le déterminent» (1940 [1937], p. 273).

Même au niveau de ce que Röpke nomme un besoin anthropologique, soit ici la propriété comme unique droit social reconnu, il ne voit rien de naturel dans son traitement et sa reconnaissance par l'individu :

Pour la propriété - à la différence du revenu - il faut un certain effort de volonté, une certaine conception de la vie qui n’est rien moins qu'évidente. [...] Chacun veut «avoir», et nulle politique révolutionnaire n’est plus populaire que celle qui veut prendre aux uns pour donner aux autres. Mais «posséder »? Cela présuppose davantage en économie, en effet : équilibre du présent et du futur, sens de la continuité et de la conservation, volonté d'être indépendant, sentiment de famille développé. Une fructueuse politique de restauration de la propriété ne débute donc point par une promesse, mais par une exigence, par un appel moral, par une œuvre éducative (1946 [1944], p. 255-256).

32 Afin de compenser l'influence des masses, Röpke propose même une capacité de suffrage différenciée selon les individus, dérogeant au principe de la démocratie moderne puisqu'il est prêt à accorder «plusieurs voix aux chefs de famille et à ceux qui se sont distingués dans leur profession.» (1946 [1944], p. 169). En ce sens, il s'inscrit dans les réactionnaires de la «deuxième vague» mise en exergue par Hirschman dans son célèbre essai Deux siècles de rhétorique réactionnaire - c'est à dire qui s'opposent au suffrage universel. II mobilise la rhétorique romantique de «l'effet pervers» (perversity) à cet effet (Hirschman, 1991, p. 18, p. 22, p. 235). 
Ici comme ailleurs, la réponse, bien que politique et économique, doit se fonder sur une formation morale si elle veut avoir du sens et être pérenne.

En fait, tout se passe comme si la souveraineté populaire se déplaçait de la sphère politique vers la sphère économique; ce qui fait sens dans la mesure où ses analyses tendent à défendre l'idée que «le marché ne soit, en fin de compte, qu’un ordre politique» (Joerges, 2006, p. 249). En effet, la souveraineté n’existe plus tellement par la représentativité ou par l'impact de la volonté populaire sur les décisions politiques ${ }^{33}$ - la genèse des normes provient d'une élite experte (Wörsdörfer, 20I2) -, mais passe directement par la capacité à faire des choix sur le marché; d'où l'exigence d'un fonctionnement impeccable de ce dernier.

La perte du choix politique, le développement des «tribunaux économiques » ou la diffusion de l'information dans la sphère marchande sont autant d'exigences qui vont dans le sens d’une démocratie économique de consommateurs ${ }^{34}$. Röpke propose comme critère normatif une certaine «souveraineté du consommateur». La première occurrence de ce concept est fournie par l'économiste libéral W.H. Hutt en 1936 - évoqué dès 1934 plus légèrement (Keizer, 1986). Comme le remarque Persky (1993, p. I84), Röpke parle dans son article de 1935 de «démocratie des consommateurs», il est vrai sans lui donner un contenu identique et surtout aussi précis que ne le fera Hutt. Favoriser l'intérêt général, chez Röpke, c'est favoriser l'intérêt du consommateur au détriment de l'intérêt privé du producteur. Mais en pratique, ce dernier l'emporte bien souvent sur l'intérêt des consommateurs en raison de causes psychologiques:

33 Bruno Amable fait justement remarquer que «cette limite à la souveraineté populaire est un thème capital de la pensée néo-libérale» dans son ensemble (Amable, 2011, p. 17, notre traduction).

34 Comprise comme une démocratie qui se réalise non pas dans le choix politique mais économique. Röpke ne cesse de filer la métaphore de la monnaie qui s'exprime comme autant de bulletins de votes, réalisant par les décisions d'achat et de vente un «plébiscite de tous les jours» (1940 [1937], p.246-247, p.274; 1942, p.253; 1945 [1942], p. 137-138). II relève néanmoins une difficulté, «d'ailleurs corrigible dans une certaine mesure -: les bulletins de vote sont distribués très inégalement. Mais ce défaut est en partie compensé par l'avantage d'une représentation proportionnelle parfaite: les minorités ne sont pas écrasées par les majorités et chaque bulletin de vote est valable. Nous avons ainsi une démocratie du marché qui, dans sa précision implicite, surpasse la démocratie politique la plus parfaite. Voici donc "l'économie planifiée" de l'économie du marché pur» (1945 [1942], p. 137-138). 
En effet, comme producteur, chaque homme est, en vertu de la division du travail, intéressé à une rareté aussi grande que possible d'une certaine marchandise, tandis que l'intérêt que chaque homme a, en tant que consommateur, à la rareté aussi petite que possible est réparti sur d'innombrables marchandises. De la sorte, le jugement de chaque individu en matière économique est déterminé plus par sa position de producteur que par sa position de consommateur et l'intérêt toujours concentré du producteur a beau jeu contre l'intérêt dispersé du consommateur (I940 [1937], p. II9).

Seul le mécanisme de la concurrence est à même de neutraliser l'expression du pouvoir sur le marché, il confère aux consommateurs la capacité d'orienter et de déterminer l'offre productive. En plus des principes qui régissent le corps de l'État, une réflexion est également engagée sur le type d'organisation hiérarchique qui doit être mise en place dans un pays pour endiguer la démocratie de masse moderne.

Röpke soutient une décentralisation se rapprochant du fédéralisme et allant jusqu'au principe de subsidiarité ${ }^{35}$. Ces principes vont dans le sens d'une responsabilité accrue des individus afin d'éviter que «le centre de gravité des décisions et des responsabilités se déplace de plus en plus vers le sommet de la pyramide sociale » (I96I [1958], p. 29), tout en ménageant l'espace de décisions réservé à l'élite, au sein d'une communauté nivelée par une «échelle naturelle de degrés » (I946 [1944], p. I55). Mais l'aspect primordial de politique publique consiste en une volonté de déconcentrer la propriété foncière (dans un sens interindividuels mais également géographique), ce qu’il nomme une «plani-

35 «Ainsi conçu, le principe de la décentralisation politique revêt une signification vaste et générale qui déborde le principe du fédéralisme et qui serait caractérisée au mieux par une expression de la doctrine catholique, le "principe de hiérarchie". C'est-à-dire qu'en partant de chaque individu et en remontant jusqu'à la centrale étatique, le droit originel se trouve dans l'échelon inférieur et chaque échelon supérieur n'entre en jeu subsidiairement à la place de l'échelon immédiatement inferieur que lorsqu'une tâche excède le domaine de ce dernier. Ainsi se constitue un échelonnement de l'individu, par delà la famille et la commune, jusqu'au canton et, finalement, jusqu'à l'État central, échelonnement qui, à la fois, limite l'État même à qui il oppose le droit propre des échelons inferieurs avec leur sphère inviolable de liberté» (1946 [1944], p. 16I). L'idée de subsidiarité provient directement de la doctrine sociale de l'église, la forme que l'on connait actuellement a été théorisée au XIXe siècle. C'est un principe normatif qui cherche à établir de l'ordre, une harmonie et une cohérence au sein d'une société. II n'est pas propre à l'État mais bien à toute forme de pouvoir et d'autorité. Pour un aperçu exhaustif, on peut consulter l'ouvrage de Delsol (1992). 
fication sociologiquement juste du pays » (ibid., p. 250). Elle s'exprime négativement par des obstacles à l'agrandissement et à l'agglomération (par exemple avec une limite dans la population des villes portée à 60000 habitants) et positivement par l'accroissement de logements de famille avec jardin, réunissant ainsi propriété d'habitation et de production de première nécessité.

\subsection{INTERVENTIONNISME LIBÉRAL ET CONSERVATISME SOCIOLOGIQUE}

Le nouveau programme du libéralisme prend chez Röpke plusieurs dénominations: il parle de «libéralisme constructif», ou «humanisme économique», mais préfère le terme de «tiers chemin» ou de «troisième voie» (dritte Weg) (1940 [1937], p. 24; 1945 [1942], p. 43; 1946 [1944], p. 38). Ce tiers chemin passe par trois objectifs: I) une décentralisation radicale (précédemment évoquée) ; 2) des règles orientant le rôle économique de l'État; 3) l'encouragement des modes de vie sociologiquement sains. Ces deux derniers objectifs sont l'objet de cette partie finale.

On tâchera également de souligner toute l'ambivalence du libéralisme röpkéen mariant un conservatisme symptomatique dans le domaine social à une vision radicalement moderne de l'intervention étatique. Dans tous les cas, cette nouvelle voie passe par un volontarisme exacerbé au profit d'«un ample programme de rigoureuse politique économique positive, avec une liste importante de tâches à accomplir» (1946 [1944], p. 65).

Outre le cadre juridico-légal, la mise en place d'institutions et le travail de conversion morale - qui relèvent d'une dimension extra-économique - venant à l'appui d'une concurrence efficace, Röpke propose deux types de critères pour juger de la pertinence d'une intervention de la part de l'État au sein même du processus économique. Il faut distinguer entre les interventions d’adaptation et celles de conservation, ainsi qu'entre mesure conforme et non-conforme. Le second critère étant chronologiquement le premier mis en exergue par l'auteur, il y revient continuellement (1936b, p. 337; 1936a, p. 195; 1940 [1937], p. 268; I945 [1942], p. 206-208; 1946 [1944], p. 68). 
Les interventions de conservation sont dangereuses et réactionnaires, elles doivent être à tout prix évitées. Seules les interventions d'adaptation - allant dans le sens de l'évolution des structures de marché, les accompagnant, facilitant et accélérant certains changements - doivent être promulguées :

Nous appuyons les secondes pour adoucir les duretés et les conflits des permutations et des entraves dans la vie économique, pour aider des groupes faibles dans leur lutte pour la vie, bref, pour agir conformément au sens de l'économie de marché autant qu'au simple commandement de la raison et de l'humanité. Et, pour le dire plus expressément, nous considérons que certains domaines de la vie économique: agriculture, métiers, artisanat, cercle des ouvriers et employés, apparaissent particulièrement faibles, menacés et dignes, pour des raisons de principes, d'une aide de ce genre (1946 [1944], p. 65).

En outre, les interventions sont conformes lorsqu'elles respectent «la structure interne de notre système économique [laissant] le mécanisme de marché intact et [atteignant] leurs objectifs non pas en contrevenant aux règles de ce mécanisme, mais en s'appuyant sur elles» (I936a, p. I95). En somme, une intervention est dite conforme lorsqu'elle n'en appelle pas d'autres et qu'elle laisse intact le mécanisme de formation des prix. Röpke met en garde l'État contre toute sur-intervention de sa part en rappelant que ce n'est pas parce qu'une action est conforme qu'il faut nécessairement en user : «La conformité est simplement une condition nécessaire, non une condition suffisante, pour une intervention à effectuer; elle indique l'instrument le plus approprié, mais non pas le but lui-même. » (1946 [1944], p. 68).

À tire d'exemple, il propose comme interventions conformes la politique douanière, la dévaluation monétaire, une politique de redistribution mesurée $^{36}$, la nationalisation ou encore le jour de repos le dimanche. En opposition, il rejette catégoriquement le contrôle des devises, les politiques de quotas à

36 Laquelle semble plutôt ambitieuse en comparaison de ce que d'autres néolibéraux proposeront d'autant qu'elle est couplée à une politique fiscale (impôt progressif, taxation importante de l'héritage etc.). Röpke expose cette recherche pour plus de justice dans la répartition de la façon suivante: «I) le changement "organique" de la répartition du revenu par catégorie; 2) le changement de la répartition personnelle du revenu et 3) la correction de la répartition - hors de l'économie - du revenu » (1940 [1937], p. 248-25I). Néanmoins, cette politique sociale n'est qu'une partie de la politique - bien plus vaste - sociologique (Bilger, 1964, p. 107). 
l'importation ou contrôle des échanges, les fixations de prix ou les accords de compensation (clearing). De son point de vue, chacune de ces mesures s'avère «susceptible d'engendrer une chaîne de répercussions menant à toujours plus d'interventions radicales, jusqu'à ce qu'on arrive en bout de chaîne à une économie de pur collectivisme» (1936b, p. 337).

Il ajoute enfin une politique de structure, laquelle occupe une «place importante et même dominante [de ce] programme», elle repose sur une action perpétuelle à l'endroit des conditions sociales préalables et résultantes de l'économie de marché : c'est à dire la «répartition des revenus et de la propriété, [la] grandeur de l'exploitation, [la] répartition de la population entre villes et campagne, entre industrie et agriculture et entre les différentes classes » (1946 [1944], p. 69).

Revenons maintenant sur un cas concret et fondamental, transversal à l'ensemble des différentes politiques étatiques: le monopole. Nous l'avons vu, le problème que pose le monopole est autant social qu'économique; «le monopole n'est pas seulement socialement injustifiable, mais il représente aussi un corps étranger dans le processus économique et un frein de la productivité totale» (1945 [1942], p. 300). Sociologiquement et économiquement néfaste, la firme monopolistique va même jusqu'à saper les fondements des valeurs tutélaires prônées par le libéralisme: «La propriété trouve sa justification, difficilement réfutable, sous le régime de la concurrence, mais elle la perd sous le régime des monopoles. » (ibid., p. 239).

Aussi, une législation ${ }^{37}$ contraire à l'instauration des monopoles doit-elle être défendue. Mais, surtout, un contrôle assidu et permanent doit être exercé par un office indépendant. Est-ce à dire qu'il pense pouvoir obtenir une économie affranchie de toute tendance monopolistique ${ }^{38}$ ? Classiquement, il reconnaît

37 L'État doit refuser les avantages précédemment accordés (attitude passive) et avoir une politique délibérément anti-monopolistique (attitude active) qui s'attaque aux autres causes (par exemple la publicité). Selon Röpke, il faut s'inspirer du Sherman Antitrust Act (1890) et aller plus loin en recourant à une politique cohérente avec cette règle de droit, contrairement à l'exemple américain (1945 [1942], p. 306). Des propositions vont dans ce sens dès 1934: par exemple avec A Positive Program for Laissez Faire, Some Proposals for a Liberal Economic Policy de H. C. Simons (195I [1934]).

38 Röpke évoque à l'occasion les travaux de Chamberlain et Robinson sur la concurrence «mono- 
l'existence de monopoles naturels (services publics, transports, électricité, etc.) et demeure très méfiant vis-à-vis de l'exploitation privée des ressources naturelles, soutenant qu'on ne pourra compter sur une économie raisonnable dans ce domaine : il souhaite se prémunir de toute «exploitation rapace des trésors naturels » (1940 [1937], p. 291, note).

Dans un premier temps, Röpke s'oriente vers la nationalisation de l'ensemble des monopoles privés pour conserver uniquement des monopoles publics (1945 [1942], p. 300). Mais dans son ouvrage suivant, on ne retrouve plus cette idée, la position apparaît comme moins radicale puisqu'il n'envisage la nationalisation que «si la surveillance officielle ne suffit pas» (1946 [1944], p. 274). En pratique, un monopole est toléré tant qu'il propose un prix comparable à une situation de concurrence, soit une tarification au coût marginal. Mais l'action n'est pas ciblée uniquement sur le producteur, l'acheteur n’est pas en reste puisque l'«éducation du consommateur et une propagande intelligente et efficace peuvent affaiblir considérablement la puissance des monopoles» (1945 [1942], p. 307).

Toutes ces mesures précédemment évoquées - en amont, pendant le processus et enfin en contradiction des résultats - font du libéralisme röpkéen un interventionnisme diffus, permanent et total, mais circonstancié par des règles d’application:

Marché libre et concurrence [...] ne résultent pas de la conduite purement passive de l'État, [...] ils ne sont donc pas le résultat surprenant et positif d'une politique économique négative. Ils représentent au contraire un résultat artificiel, fragile et dépendant d’une infinité de conditions qui ne réclament pas seulement une éthique économique très élevée, mais également un gouvernement qui, par législation, son administration, sa jurisprudence, sa politique financière et sa direction morale et spirituelle, travaille en faveur du maintien de la liberté de marché et de la concurrence, en créant le cadre institutionnel et juridique, en déterminant les règles de la lutte économique et en surveillant leur application avec une sévérité toute impartiale, mais impitoyable (ibid., p. 299).

polistique» ou «imparfaite» qu'il trouve fort intéressant, se félicitant même des développements futurs que peut engendrer un tel programme de recherche (1942, p. 229). Pour autant, il se situe sur une base de compréhension totalement différente et ne tient pas réellement compte de ces analyses: «le fait que la libre concurrence n'existe pour ainsi dire pas dans la réalité à l'état chimiquement pur et que la plupart des prix contiennent un élément monopoliste, ne doit pas faire naître l'opinion erronée que notre système économique au fond ne repose plus sur la concurrence, mais sur le monopole.» (1940 [1937], p. 203). 
Mais cette approche radicalement moderne du rôle de l'État dans l'économie doit être mise en parallèle avec le conservatisme sociologique de l'auteur, soulignant un autre aspect de son interventionnisme englobant.

Sa vision de la société le conduit à mettre sur un piédestal deux catégories socioéconomiques : les paysans et - dans une moindre mesure - les artisans. Il est intéressant de noter que l'école de Fribourg a pu être rapprochée de l'école Physiocrate française, sous l'aspect sociologique de la relation maître à élèves (Quesnay/Eucken), mais également à travers leur commune volonté d'une gouvernance par les experts ou de conseil aux Princes (Bilger, 2003 ; Commun, 2009). L'exemple de Röpke peut accréditer, sur le fond, cette comparaison dans la mesure où il accorde une place éminente à l'agriculture. Mais loin d'en faire la seule classe productrice de valeur économique, il opère un glissement et fait du monde paysan le seul producteur d'une moralité saine; parlant de véritable «colonne vertébrale d'une nation saine» (1945 [1942], p. 263). Le mode de vie paysan représente l’idéal sociologique röpkéen:

C’est moins la production de denrées alimentaires et de matières premières organiques comme telle qui nous intéresse ici que cette forme particulière de la production que nous appelons paysanne, car elle présente encore cet aspect sociologique si typique qui nous importe comme point de départ (ibid., p. 262).

À l'instar du domaine de l'entreprise, on ne saurait promouvoir de grandes exploitations ou la concentration des propriétés terriennes. Il faut favoriser la production de denrées agricoles de haute qualité, des cultures harmonieuses et diversifiées. Les règles sont ici claires et restrictives, contrevenant même à une pure logique d'économie de marché concurrentielle:

L’une des caractéristiques essentielles [...] est qu'une seule exploitation ne doit pas dépasser en importance le domaine qui peut être travaillé par une famille et des domestiques, journaliers auxiliaires et saisonniers; dans la règle, le domaine appartient au paysan et incarne la tradition, chaîne des générations ; il est intégré dans l'association sociale de la famille, du village, des associations coopératives, et dans la communauté de la profession et du voisinage, étrangère à toute pensée de concurrence; obéissant au circuit de la nature et à ses lois, il représente une exploitation mixte [...]. L'exploitation agricole paysanne [...] est donc le complément normal du côté industriel et citadin de notre civilisation, lui opposant la tradition et l'enracinement terriens, l'indépendance économique et l'auto- 
approvisionnement, la proximité de la nature, la mesure et la sérénité, une existence naturelle près des sources de la vie, et une incorporation consentie dans la chaîne de la vie et de la mort (ibid. p. 263-264, souligné par nous).

Le monde paysan fonctionne comme une véritable force sociologique antagoniste à la tendance naturellement déstructurante du capitalisme concurrentiel et demeure un point névralgique du programme de l'auteur, une forme de libéralisme communautaire contra-capitaliste.

Bien entendu, le mode de vie paysan ne peut être généralisé à l'ensemble du corps social. Si le monde de l'artisanat s'en rapproche, notamment par les conditions de propriété, tout doit être entrepris pour que la classe ouvrière et les employés tendent vers cet idéal. Pour sortir de cette ère des masses, il conviendra de proposer à l'ouvrier industriel :

[...] un genre de vie et un mode de travail si possible analogues à ceux du paysan et de l'artisan. Cette politique suppose en tout premier lieu, et comme règle fondamentale, une décentralisation des industries à la campagne et dans les petites villes et un plan d’aménagement national luttant contre l'entassement industriel dans les grandes cités (ibid., p. 286).

L'auteur déploie à ce sujet une vision radicalement antimoderne et conservatrice. Si son libéralisme dépasse le naturalisme et le romantisme du «Laissez faire» dans sa revendication d’un interventionnisme économique conséquent, il retombe dans ce tropisme par sa conception surannée de l'homme qui passe par l'éloge des «structures sociales précapitalistes» (Ptak, 2009, p. I23, notre traduction) ou l'affirmation de certaines valeurs morales prétendument immuables. Il fait finalement peu de cas de la liberté politique, recherche une liberté économique nécessaire et indispensable, mais signale qu'elle reste non suffisante (i940 [1937], p. 23 ; I942, p. 263; I945 [1942], p. 23I), laissant place à l’élection conservatrice de certaines classes morales qui repose sur un profil professionnel (que ce soit les clercs ou le monde agraire). Comme le notait justement Bilger (2003, p. 25), l'idéalisme communautariste ${ }^{39}$ de Röpke ne reconnaît pas la liberté comme «bien suprême»; à la différence de nombreuses mouvances (néo-)libérales. 
La notion de propriété est centrale dans le domaine économique comme sociologique; nous remarquons d'ailleurs que sa résolution en faveur d'une répartition plus équitable apparaît somme tout comme progressiste, tout du moins à forte préoccupation sociale. La propriété privée devient même le vecteur «d'une liberté sociale et morale» (Bilger 1964, p. I08) en donnant à chacun les moyens d'exercer ses potentialités ainsi qu'une indépendance relativement aux effets négatifs de l'économie de marché ${ }^{\circ}$. Ce faisant, il inscrit définitivement son libéralisme dans un espace bien plus vaste que le seul libéralisme économique ou politique.

En définitive, l'articulation entre ses critères de politiques économiques d'un côté et ses principes sociologiques normatifs de l'autre pose question. En effet, comment favoriser une mesure d'adaptation au détriment d'une politique de conservation tout en respectant la volonté liminaire de préserver la forme figée de l'existence agrarienne? À notre sens, si une mesure d'adaptation tend vers une politique de préservation artificielle de cet idéal social röpkéen, elle amène fatalement à détruire toute la cohérence interprétative dudit critère. Un tel mariage semble cohérent seulement si l'auteur accepte une philosophie de l'histoire proprement téléologique et déterministe, ce qu'il n'envisage à aucun moment. Au demeurant, cette tension s'accompagne d'une incohérence discursive, puisque Röpke s'adresse à deux types de populations non superposables. Comme sociologue, il perpétue une tradition de lecture classique de la société, en terme de classes sociales, par opposition au vocabulaire microéconomique mobilisé à d'autres moments de l'analyse.

aspirations, des habitudes ou idéaux semblables (i.e. bien souvent une conception en terme de minorités) qu'elle recouvre une définition géographique et sociale.

40 Cette vision de la liberté dans le domaine moral et social peut être rapportée au concept de liberté positive; c'est à dire à la place éminente accordée à la capacité de choix et d'action d'un individu au sein de la collectivité (voir Berlin, 1969 [1958]). On trouve les bases d'une telle vision chez Röpke dans une conférence donnée en 1933 (1933a, p. 83-93). Bien que plus cohérente et plus ambitieuse que la seule liberté négative (protection contre toute coercition extérieure), Röpke semble néanmoins tomber dans les travers d'une telle approche: «les détracteurs de la liberté positive ont fait valoir qu'elle dégénère en un concept qui peut être utilisé pour soutenir un élitisme autoritaire, où la coercition jugée bonne pour la personne contrainte est nommée la liberté» (McCloskey 1974, p. 15, notre traduction). 


\section{CONCLUSION}

Si nous avons pris au sérieux l'analyse historique de Wilhelm Röpke dans la première partie de ce travail, ce n'est pas dans l'optique d'une recherche de validité interne ou externe. Plutôt, cette analyse contingente et souvent contestable nous intéresse en ce quelle préfigure tout en l'éclairant la proposition d'un renouveau au sein de la pensée libérale, à savoir tout un pan de l'ordolibéralisme allemand.

Ce travail a cherché à dénouer les différentes catégories de causes du déclin économique et social de l'Occident proposées par Wilhelm Röpke. Ce faisant, il a permis de construire la symétrie entre ces causes et les solutions par lesquelles il entend y remédier. Ce premier pas permet de dégager l'herméneutique historique de l'auteur, laquelle s'insère parfaitement dans l'une des catégories de l' «idéologie», telle qu'identifée par Paul Ricœur : elle représente fondamentalement une «théorie de la motivation sociale» dont le «rôle médiateur reste irremplaçable; il s'exprime par ceci que l'idéologie est toujours plus qu'un reflet, dans la mesure où elle est aussi justification et projet» (Ricœur, 1974, p. 332).

Par ailleurs, en rendant explicite son analyse du déclin, il devient évident que l'ordolibéralisme - dans sa version röpkéenne - ne pouvait pas se contenter d'apporter des réponses dans le seul domaine économique. Il a donc embrassé des conceptions morales et politiques cherchant à résorber en profondeur les causes identifiées, et non traiter exclusivement les symptômes. En nous attachant à mettre à jour les nombreux déterminants extra-économiques au cœur de sa pensée et qui renforcent le caractère singulier de ses analyses, nous avons mis en évidence que le tiers chemin que Röpke appel de ses vœux reste prisonnier d'une tension perpétuelle entre progressisme économique d'un côté et conservatisme social et moral de l'autre.

NDA. Ce travail a bénéficié de multiples remarques recueillies au sein de séminaires à Lausanne (HIPE) et à Paris $(\mathrm{AOH})$. Je tiens à remercier chaleureusement tous ses participants. Merci également au secrétaire de rédaction de la Revue européenne des sciences sociales pour sa relecture attentive et ses remarques qui m'ont permis d'améliorer le texte. Toute erreur ou maladresse est mienne. 


\section{BIBLIOGRAPHIE}

AMABLE Bruno, 20II, «Morals and Politics in the Ideology of Neo-liberalism», Socio-Economic Review, 9-I, p. 3-30.

AUDIER Serge, 2008, «La face cachée du "Néo-libéralisme"» in Id., Aux origines du néo-libéralisme: Le colloque Lippmann, Lormont, Éditions Le Bord de l'eau, p. 7-245.

-, 2012, Néolibéralisme(s), Paris, Grasset.

BERLIN Isaiah, 1969 (1958), «Two Concepts of Liberty» in Id., Four Essays on Liberty, Oxford, Oxford University Press, p. 118-172.

BILGER François, 1964, La Pensée économique libérale dans l'Allemagne contemporaine, Paris, Librairie générale de droit et de jurisprudence.

-, 2003, «La pensée néolibérale française et l'ordolibéralisme», in Commun, 2003a, p. 17-30.

BOARMAN Patrick M., 2000, «Wilhelm Röpke: Apostle of a Humane Economy», Society, 37-6, p. 57-65.

BÖHM Franz, EUCKEN Walter et GROSSMAN-Doerth Hans, 1989 (1936), «The Ordo Manifesto of 1936», in Germany's social market economy: Origins and evolution, Alan Peacock et Hans Willgerodt (dir.), London, Macmillan for the Trade Policy Research Centre, p. 15-26.

BONEFELD Werner, 2012, «Freedom and the Strong State: On German Ordoliberalism», New Political Economy, 17-5, p. I-24.

-, 2013, «Human Economy and Social Policy: On Ordo-Liberalism and Political Authority », History of the Human Sciences, 26-2, p. 106-125.

BROYER Sylvain, 2007, «La pensée théorique et politique de Walter Eucken à la lumière des écoles historiques allemandes», Thèse de doctorat, Lyon-2 Lumière.

BRUHNS Hinnerk, 2004, Histoire et économie politique en Allemagne de Gustav Schmoller à Max Weber: nouvelles perspectives sur l'école historique de l'économie, Paris, Éditions de la MSH.

BURGIN Angus, 2012, The Great Persuasion: Reinventing Free Markets since the Depression, Cambridge, Mass., Harvard University Press. 
CAMPBELL William F., 2002 (1995), «Wilhelm Röpke and the City of Man », in Wilhelm Röpke, The Moral Foundations of Civil Society, New Brunswick, Transaction Publishers, p.ix -xxvi.

- et FOSHEE Andrew W., 1997, «Catholic Social Encyclicals and Wilhelm Röpke's Political Economy of the "Third Way"», Catholic Social Science Review, 2, p. I|7-144.

CLAVE Francis Urbain, 2005, «Walter Lippmann et le néolibéralisme de La Cité Libre». Cahiers d'économie Politique, 48-I, p.79-110.

COLEMAN William, 2013, «What Was "New" About Neoliberalism?», Economic Affairs, 33-I, p. 78-92.

COMMUN Patricia, 2003a, L'Ordolibéralisme allemand: aux sources de l'économie sociale de marché, Patricia Commun (dir.), Cergy-Pontoise, Travaux et documents du C.I.R.A.C.

-, 2003b, «La conversion de Ludwig Erhard à l'ordolibéralisme (1930-1950)», in Commun, 2003a, p. 175-199.

-, 2009, «Walter Eucken entre économie et politique (|89|-1950) 》, in La Pensée économique allemande, Alain Alcouffe et Claude Diebolt (dir.), Paris, Economica, p. 375-398.

-, 2014, «German Ordoliberalism: Order Vs. Disorder In Röpke's Early Works », i-lex, 21, p. 27-39.

DELSOL Chantal, 1992, L'État subsidiaire : ingérence et non-ingérence de l'État, le principe de subsidiarité aux fondements de l'histoire européenne, Paris, PUF.

DENORD François, 200I, «Aux origines du néo-libéralisme en France», Le Mouvement social, 195-2, p.9-34.

DOERING Detmar, 2006, «Wilhelm von Humboldt et les origines du libéralisme allemand », in Histoire du libéralisme en Europe, Philippe Nemo et Jean Petitot (dir.), Paris, PUF, p. 859-880.

EUCKEN Walter, 1950 (1940), The Foundations of Economics, trad. Terence W. Hutchison, London-Edinburgh-Glasgow, W. Hodge.

-, 1948, «On the Theory of the Centrally Administered Economy: An Analysis of the German Experiment», Part I \& II, Economica, New Series, 15-58, p. 79-100 et 15-59, p. 173-193. 
FÈVRE Raphaël, 2015, «Du libéralisme historique à la crise sociale du Xxe siècle: la lecture de Wilhelm Röpke», Revue économique, 66-5, p. 90I-932.

FISHER Irving, 1907, «Why the Doctrine of Laissez faire Been Abandoned?», Science, 25-627, p. $18-27$.

FOUCAULT Michel, 2004a, Naissance de la biopolitique, Paris, Seuil/Gallimard.

-, 2004b, Sécurité, Territoire, Population, Paris, Seuil/Gallimard.

FRIEDRICH Carl J., 1955, «The Political Thought of Neo-Liberalism», The American Political Science Review, 49-2, p. 509-525.

GERBER David J., 1994, «Constitutionalizing the Economy: German Neo-liberalism, Competition Law and the "New" Europe», American Journal of Comparative Law, 42, p. 25-84.

-, 1998, Law and Competition in Twentieth Century Europe: Protecting Prometheus, Oxford, Clarendon Press.

GOLDSCHMIDT Nils, 2006, «Alfred Müller-Armack et Ludwig Erhard: Le libéralisme social de marché», in Histoire du libéralisme en Europe, Philippe Nemo et Jean Petitot (dir.), Paris, PUF, p. 953-983.

GREGG Samuel, 2010, Wilhelm Röpke's Political Economy, Cheltenham (UK), Northampton (Mass.), Elgar.

GROSSEKETTLER Heinz G., 1989, «On Designing an Economic Order. The Contributions of the Freiburg School, in Twentieth-Century Economic Thought, Donald A. Walker (dir.), II, Aldershot Brookfield, Elgar, p. 38-84.

-, 1994, «On Designing an Institutional Infrastructure for Economies: The Freiburg Legacy after 50 Years», Journal of Economic Studies, 21-4, p. 9-24.

HABERMAS Jürgen, 1989, The New Conservatism: Cultural Criticism and the Historian's Debate, Cambridge (Mass.), Polity Press.

HANDMAN Max et al., 193I, «Economic History - The Decline of Laissez Faire (Round table conferences)», The American Economic Review, 21-I, p. 3-I0.

HAYEK Friedrich A., 1948, «Individualism: True and False» in Id., Individualism and Economic Order, Chicago, The University of Chicago Press, p. I-32.

-, 194I, «The Counter-Revolution of Science», Economica, 8-31, p. 28I-320. 
HENNECKE Hans-Jörg, 2005, Wilhelm Röpke - Ein Leben in der Brandung, Stuttgart, Schäffer-Poeschel.

HIRSCHMAN Albert O., 1991 (1991), Deux siècles de rhétorique réactionnaire, trad. Pierre Andler, Paris, Fayard.

HUTCHISON Terence W., 1979, «Notes on the Effects of Economic Ideas on Policy: the Example of the German Social Market Economy», Zeitschrift für die gesamte Staatswissenschaft, |35-3, p.426-44|.

JACKSON Ben, 2010, «At the Origins of Neo-Liberalism: The Free Economy and the Strong State, 1930-1947», The Historical Journal, 53-I, p. 129-I5I.

JOERGES Christian, 2006, «La constitution économique européenne en processus et en procès», Revue internationale de droit économique, 20-3, p. 245-284.

KEIZER Willem, 1986, The Concept of «Consumer» Sovereignty. Exposition, Critique, Defense, Serie Research Memoranda 28, VU University Amsterdam.

KEYNES John Maynard, 1999 (1926), The End of Laissez-faire, suivi de Suis-je un libéral? trad. Frédéric Cotton, Marseille, Montréal, Agone / Comeau \& Nadeau.

KLAUSINGER Hansjörg, 1999, «German Anticipations of the Keynesian Revolution? The Case of Lautenbach, Neisser and Röpke», The European Journal of the History of Economic Thought, 6-3, p. 378-403.

KOLEV Stefan, 20 I0, «Hayek as an Ordo-Liberal», HWWI Research Paper, 5-II, p. I-22.

LAGUEUX Maurice, 1988, «Le néo-libéralisme comme programme de recherche et comme idéologie», Cahiers d'économie politique, L'Harmattan, 16/17, p. 129-152.

«Le Colloque Walter Lippmann - Institut International de Coopération Intellectuelle. Paris, le 26-30 août 1938», in Audier, 2008a, p. 245-354.

LEEN A. R., 2003. «Röpke Revisited From a Modern-Austrian Perspective: the Moral Heart of Capitalism», Economic Policy in an Orderly Framework: Liber Amicorum for Gerrit Meijer, Jurgen G. Backhaus et. al. (dir.), Münster, Lit Verlag, p. 27I-283.

LEVY David M. et PEART Sandra J., 2008, «Socialist Calculation Debate», in The New Palgrave Dictionary of Economics, Steven N. Durlauf, Lawrence E. Blume (dir.), Basingstoke, Nature Publishing Group, p. 685-692.

LIPPMANN Walter, 1946, La Cité libre, Paris, Librairie de Médicis. 
MANNHEIM Karl, 2009 (1984), La Pensée conservatrice, trad. Jean-Luc Evard, Meaux, Éditions de la revue Conférence.

MCCLOSKEY H. J., 1974, «Liberalism», Philosophy, 49-187, p. 13-32.

MEGAY Edward N., 1970, «Anti-Pluralist Liberalism: The German Neoliberals», Political Science Quarterly, 85-3, p. 422-42.

NICHOLLS Anthony James, 1994, Freedom With Responsability: the Social Market Economy in Germany, 1918-1963, Oxford, Clarendon Press.

OLIVER Henry M., 1960, «German Neoliberalism », The Quarterly Journal of Economics, 74-I, p. II7-149.

PERSKY Joseph, 1993, «Retrospectives: Consumer Sovereignty», The Journal of Economic Perspectives, 7-I, p. |83-191.

PTAK Ralf, 2009, «Neoliberalism in Germany», in The Road from Mont Pèlerin: The Making of the Neoliberal Thought Collective, Philip Mirowski et Dieter Plehwe (dir.), Cambridge (Mass.), Harvard University Press, p. 98-138.

PUHLE Hans-Jurgen, 1978, «Conservatism in Modern German History», Journal of Contemporary History, 13-4, p. 689-720.

RICCEUR Paul, 1974, «Science et idéologie», Revue Philosophique de Louvain, 72-14, p. 328-356.

ROBBINS Lionel, 1947, La Nature et la signification de la science économique, Paris, Éditions politiques économiques et sociales.

RÖPKE Wilhelm, 1933a, «End of an Era? Lecture delivered at Frankfurt am Main, February 8, 1933», in Röpke, 1969, p. 79-97.

-, 1933b, «Trends in German Business Cycle Policy», The Economic Journal, 43- 17I, p. 427-44I.

- 1935, «Fascist Economics», Economica, 2-5, p. 85- 100.

-, 1936a, Crises and Cycles, Edingurgh-London-Glasgow, W. Hodge.

-, 1936b, «Socialism, Planning, and the Business Cycle», Journal of Political Economy, 44, p. 318-338.

-, 1940 (1937), Explication économique du monde moderne, Paris, Librairie de Médicis.

-, 1942, International Economic Disintegration, Edingurgh-London-Glasgow, W. Hodge. 
-, 1945 (1942), La Crise de notre temps, Neuchâtel, La Baconnière.

-, 1946 (1944), Civitas humana, Paris, Librairie de Médicis.

-, 1947, La Fonction de la petite et moyenne entreprise dans l'économie publique,

Publications de l'Union suisse des arts et métiers, 32, Berne.

-, 1956, «The Place of Economics Among the Sciences», in On Freedom and Free Enterprise: Essays in Honor of Ludwig von Mises, Mary Sennholz (dir.), Princeton, Van Nostrand, p. III-127.

-, 1957, Welfare, Freedom and Inflation, London, Pall Mall Press.

-, 1959a, L'Économie mondiale aux XIXe et Xx siècles, Genève, Droz.

-, 1959b, «The Economic Necessity of Freedom», Modern Age, 3-3, p. 227-336.

-, 1961 (1958), Au-delà de l'offre et de la demande, Paris, Payot.

-, 1964, «European Prosperity and Its Lessons», South African Journal of Economics, 32-3, p. 187-198.

-, 1969, Against the Tide, Chicago, H. Regnery.

-, 1987 (195I), «The Problem of Economic Order», in Id., Two essays by Wilhelm Röpke, Johannes Overbee (éd.), New-York, University Press of America. RUEFF Jacques, 1967 (1945), L’Ordre social, Paris, Génin.

SALLY Razeen, 1996, «Ordoliberalism and the Social Market: Classical Political Economy from Germany », New Political Economy, I-2, p. 233-258.

SIEMS Matthias et SCHNYDER Gerhard, 2012, «The Ordoliberal Variety of Neoliberalism », in Banking Systems in the Crisis: The Faces of Liberal Capitalism, London, Routledge, p. 250-268.

SIMONS Henry Calvert, I95I (1934), «A Positive Program for Laissez Faire, Some Proposals for a Liberal Economic Policy», in Economic policy for a free society, Chicago, The University of Chicago Press, p. 40-77.

SOLCHANY Jean, 2013, «Reconstituer la pensée néolibérale: le cas de Wilhelm Röpke», in «Une sociologie de la pensée politique est-elle possible? Le cas des (néo)libéralismes», section thématique 60 - Congrès de l'AFSP, Paris.

-, 2015, Wilhelm Röpke, l'autre Hayek. Aux origines du néolibéralisme, Paris, Publications de la Sorbonne. 
STEINER Yves et WALPEN Bernhard, 2006, «L'apport de l'ordolibéralisme au renouveau libéral, puis son éclipse», Carnets de bord en sciences humaines, II, p.94-I04.

WÖRSDÖRFER Manuel, 2011, «Von Hayek and Ordoliberalism on Justice», in «Justice and Economics: Ancient Doctrines and Modern Theories», Journées de l'association Charles Gide, Toulouse.

-, 2012, «Freiburg School of Law and Economics, Freiburg (Lehrstuhl-) Tradition and the Genesis of Norms», Working Paper Series, Cluster of Excellence «The Formation of Normative Orders».

WUILLÈME Tanguy, 201I, «José Ortega y Gasset: La Révolte des masses», Questions de communication, 19, p. 387-389.

YCRE Jean-Michel, 2003, «Les sources catholiques de l'ordolibéralisme allemand: Röpke et la pensée catholique sociale allemande», in Commun, 2003a, p. I63-172. 